OPEN ACCESS

Edited by: Giacomina Brunetti,

Università degli studi di Bari Aldo Moro, Italy

Reviewed by:

Antonia Sophocleous, University of Edinburgh,

United Kingdom

Melissa Orlandin Premaor,

Universidade Federal

de Santa Maria, Brazi

*Correspondence:

Kate A. Ward

kw@mrc.soton.ac.uk

tThese authors have joint senior authorship.

Specialty section:

This article was submitted

to Bone Research,

a section of the journal

Frontiers in Endocrinology

Received: 12 June 2017 Accepted: 15 August 2017 Published: 31 August 2017

Citation:

Zengin A, Fulford AJ, Sawo Y

Jarjou LM, Schoenmakers I,

Goldberg G, Prentice A and Ward KA

(2017) The Gambian Bone and

Muscle Ageing Study: Baseline Data

from a Prospective Observational

African Sub-Saharan Study.

Front. Endocrinol. 8:219.

doi: 10.3389/fendo.2017.00219

\section{The Gambian Bone and Muscle Ageing Study: Baseline Data from a Prospective Observational African Sub-Saharan Study}

\author{
Ayse Zengin ${ }^{1,2}$, Anthony J. Fulford ${ }^{3}$, Yankuba Sawo ${ }^{4}$, Landing M. Jarjou 4 , \\ Inez Schoenmakers ${ }^{1,5}$, Gail Goldberg ${ }^{1,4}$, Ann Prentice ${ }^{1,4 \dagger}$ and Kate A. Ward ${ }^{1,6 * t}$ \\ ${ }^{1}$ Nutrition and Bone Health Group, MRC Elsie Widdowson Laboratory, Cambridge, United Kingdom, ${ }^{2}$ Faculty of Medicine, \\ Nursing and Health Sciences, Department of Medicine, School of Clinical Sciences at Monash Health, Monash University, \\ Monash Medical Centre, Clayton, VIC, Australia, ${ }^{3}$ International Nutritional Group, London School of Hygiene and Tropical \\ Medicine, London, United Kingdom, ${ }^{4}$ Calcium, Vitamin D and Bone Health Group at MRC Unit The Gambia, Banjul, Gambia, \\ ${ }^{5}$ Faculty of Medicine and Health Sciences, Department of Medicine, University of East Anglia, Norwich, United Kingdom, \\ ${ }^{6}$ MRC Lifecourse Epidemiology Unit, University of Southampton, Southampton, United Kingdom
}

The Gambian Bone and Muscle Ageing Study is a prospective observational study investigating bone and muscle ageing in men and women from a poor, subsistence farming community of The Gambia, West Africa. Musculoskeletal diseases, including osteoporosis and sarcopenia, form a major part of the current global non-communicable disease burden. By 2050, the vast majority of the world's ageing population will live in low- and middle-income countries with an estimated two-fold rise in osteoporotic fracture. The study design was to characterise change in bone and muscle outcomes and to identify possible preventative strategies for fracture and sarcopenia in the increasing ageing population. Men and women aged $\geq 40$ years from the Kiang West region of The Gambia were recruited with stratified sampling by sex and age. Baseline measurements were completed in 488 participants in 2012 who were randomly assigned to follow-up between 1.5 and 2 years later. Follow-up measurements were performed on 465 participants approximately 1.7 years after baseline measurements. The data set comprises a wide range of measurements on bone, muscle strength, anthropometry, biochemistry, and dietary intake. Questionnaires were used to obtain information on health, lifestyle, musculoskeletal pain, and reproductive status. Baseline cross-sectional data show preliminary evidence for bone mineral density and muscle loss with age. Men had greater negative differences in total body lean mass with age than women following adjustments for body size. From peripheral quantitative computed tomography scans, greater negative associations between bone outcomes and age at the radius and tibia were shown in women than in men. Ultimately, the findings from The Gambian Bone and Muscle Ageing Study will contribute to the understanding of musculoskeletal health in a transitioning population and better characterise fracture and sarcopenia incidence in The Gambia with an aim to the development of preventative strategies against both.

Keywords: bone, ageing, Africa, muscle, dual energy X-ray absorptiometry, non-communicable disease, Gambia 


\section{INTRODUCTION}

Health priorities in low and middle-income countries (LMICs) have until recently focused on infectious diseases. As these populations undergo social, economic, and environmental transition, non-communicable diseases (NCDs) of older age are becoming prevalent. As life expectancy is increasing, within 35 years the vast majority of the world's older population will live in LMICs, with a consequent increase in associated NCDs $(1,2)$. The life expectancy in 2015 in The Gambia was 59.8 years in men and 62.5 years in women. In the Kiang West region, it was 65.3 and 73.5 years, respectively (3). In addition to musculoskeletal diseases, the prevalence of other diseases in adults in The Gambia include hypertension $(27 \%)$, malaria $(<2 \%), \mathrm{HIV}$, and AIDS $(1.8 \%)(3-5)$. In the current study, musculoskeletal disease is a term used to describe osteoporosis (increased risk of fragility fracture) and sarcopenia (loss of muscle mass and strength), which are major contributors to the global NCD burden (6). For example, osteoporotic fracture is expected to double in LMICs by 2040 (7). The current cohort provides a baseline from which to characterise these changes as the population undergoes transition and to determine the individual, societal, and economic impact of osteoporosis and sarcopenia, and the associated increase in falls/fragility fractures, disability, morbidity, and mortality-all of which are currently unknown.

The first study to investigate bone health in The Gambia was over 30 years ago and used single-photon absorptiometry to measure the radius of women aged 18-85 years and showed that Gambians had a 5.6\% lower bone mineral content (BMC) compared to Caucasian women (8); however, this difference was attenuated following adjustments for body size. A subsequent study in women aged $\geq 44$ years utilised dual photon absorptiometry and showed that BMC at the lumbar spine was $24 \%$ lower in Gambians compared to Caucasians following adjustments for age, weight, and height (9). At that time, the incidence of fragility fractures was reported to be rare, paralleling the current low prevalence of osteoporosis throughout sub-Saharan Africa, though it is important to note the scarcity of available data $(10,11)$. The very limited data on bone health of Gambian women utilised imaging devices that were predecessors of the current clinical gold standard, dual energy X-ray absorptiometry (DXA) (8). In addition, there are no data in Gambian men. Currently, habitual calcium intakes are low, and parathyroid hormone levels are elevated while 25-hydroxyvitamin D status is good; low calcium and high PTH are risk factors for fracture in high-risk populations (12-15). The Gambia is undergoing a transition towards Western lifestyles particularly with respect to nutrition and physical inactivity, which are likely to contribute to the predicted rise in fracture risk.

Dual energy X-ray absorptiometry is used to measure areal bone mineral density (aBMD) as a predictor of fracture risk in older people from populations at high risk of osteoporosis (16). However, the use of DXA is of limited value in different ethnicities and at different times of life $(13,17)$. Newer imaging technologies such as peripheral quantitative computed tomography (pQCT) are valuable as they can measure volumetric BMD (vBMD), cortical, and trabecular compartments separately and provide information about the structural and geometrical parameters that contribute to bone strength (17). The application of DXA combined with $\mathrm{PQCT}$ in different ethnic populations is an area of growing research that allows better understanding of the underlying determinants of bone strength.

Understanding musculoskeletal health and better characterising fracture incidence in The Gambia may help in the development of preventative strategies against the predicted rise in osteoporotic fracture and sarcopenia. Ultimately, this would improve quality of life and reduce consequent morbidity and mortality associated with the conditions in the ageing population in The Gambia. Therefore, the Gambian Bone and Muscle Ageing Study (GamBAS) cohort was established to prospectively study musculoskeletal ageing in rural Gambian men and women. The primary aim of the study was to determine change in bone mineral density in men and women, the secondary aims were to characterise change in muscle, determine fracture and sarcopenia incidence, and finally to determine the biochemical and hormonal predictors of the detected changes in bone and muscle. This manuscript presents a general cohort description and findings from the baseline data.

\section{STUDY OUTLINE}

\section{Study Design \\ Recruitment}

In 1974, the Medical Research Council United Kingdom (MRC UK) established a permanent field station in Keneba (MRC Keneba), which is located in the middle of a $750 \mathrm{~km}^{2}$ district in the Lower River Region of Kiang West (Figure 1). Research facilities in Kiang West were initially set up to support nutrition studies in four core villages which have been part of a demographic surveillance system since 1950 and were the basis for the establishment of the comprehensive demographic surveillance, the Kiang West Demographic Surveillance System (KWDSS). The KWDSS and health care provision service has expanded into the wider Kiang West community (3). The population of Kiang West was described in 2015 (3), and residents are mainly of Mandinka ethnicity (79.9\%) followed by Fula (16.2\%), Jola (2.4\%), and others (1.3\%). The GamBAS cohort was recruited from 10 villages in Kiang West (Figure 1).

All men and women aged 40 years and over and residing within the four core survey villages of Kiang West were identified using the KWDSS (Figure 2) (3). Potential participants were initially selected from the four core villages (Keneba, Manduar, Jali, and Kantong Kunda) to ensure a population representative of those who were known to have been born locally. Target group sizes were not reached; therefore, individuals from six other villages in the area were also recruited (Figure 1). Individuals were ranked according to the accuracy of date of birth ascertained from the KWDSS, from most to least accurate (that is, exact day, month or year), where recruitment priority was given to those with the most accurate. After initial village sensitisation and discussion with the elders, participants were located and approached by fieldworkers who explained the study in the local language and invited them to participate. 


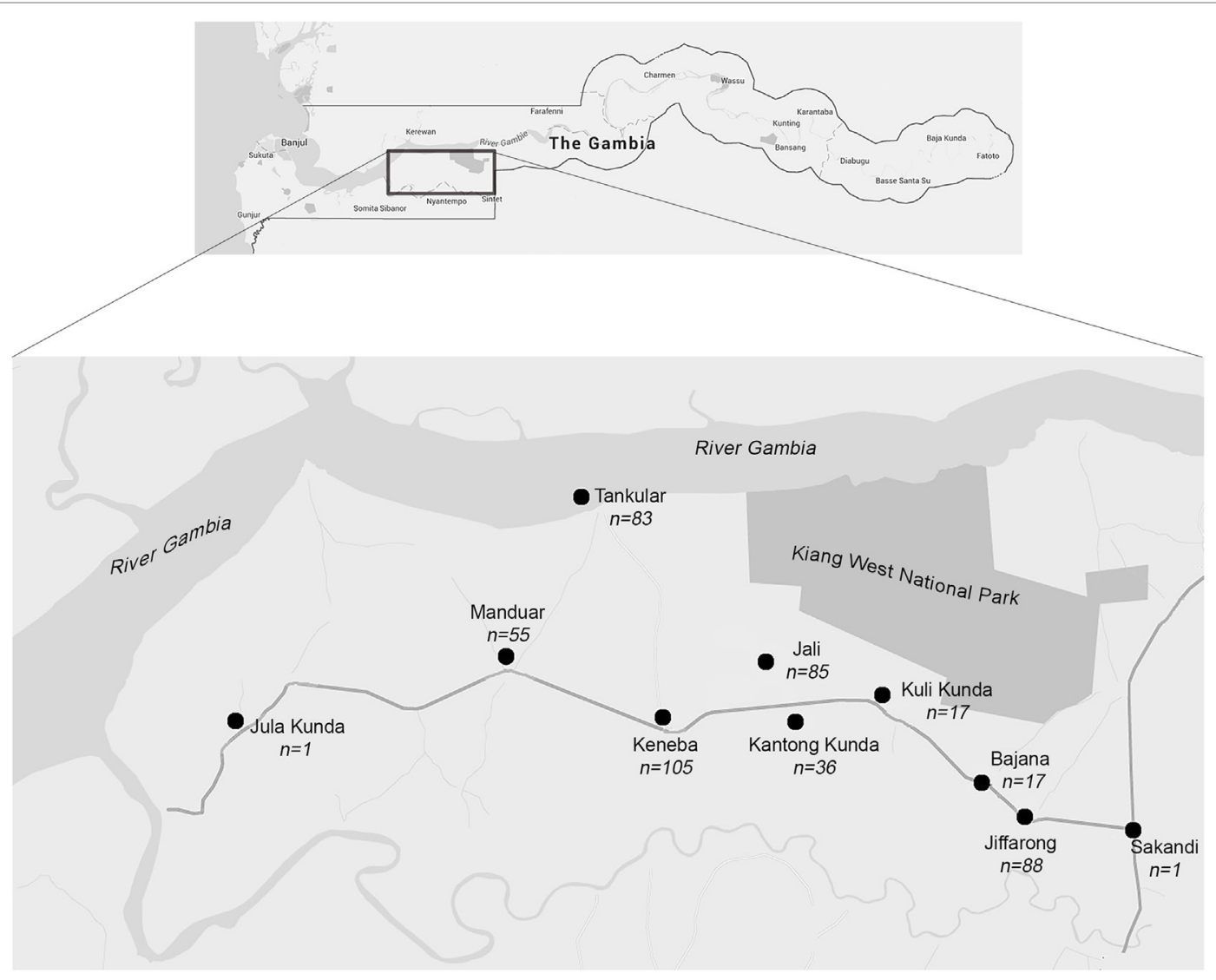

FIGURE 1 | Map of the study area. The Kiang West region is located in the lower region of The Gambia.

\section{Eligibility Criteria}

All eligible participants aged 40 years and over were given a verbal explanation of all the elements of the study and completed a screening questionnaire. If eligible, participants were given an information sheet and asked to sign or thumbprint an informed consent form and were enrolled in the study. Recruitment commenced in March 2011 and baseline measurements in 488 individuals were completed over 18 months (Figure 2).

\section{Exclusion Criteria}

Pregnant and lactating women were excluded. A woman was considered non-pregnant, non-lactating if she was at least 3 months post lactation and had regular menses. Individuals who were deemed too physically frail or incapable, due to existing disability or chronic illness to attend MRC Keneba for measurements were excluded from participating in the study (Figure 2). This was determined by the individual themselves, their spouse/relative or compound elder or a member of the fieldwork team. Prior to enrolment, participants were confirmed to not already be part of an on-going study at MRC Keneba (or elsewhere). The use of prescribed or self-medication to control pain (e.g., aspirin, paracetamol), indigestion (e.g., magnesium trisilicate, dried baobab fruit), chronic diseases of ageing (e.g., diuretics, local medicine), or use of chemical contraception (e.g., Depo Provera) was not an exclusion criterion.

\section{Follow-up}

The first follow-up measurements were scheduled 1.5-2 years later ( $1.7 \pm 0.2$ years) after the baseline measurement and were completed by the end of 2015 (Supplementary Material, Figure 2). Of the 488 participants who attended baseline visits, 380 had a repeated set of complete measurements taken. The remaining 108 participants who did not visit Keneba were approached for home visit: 85 were measured at home, 9 were away or could not be reached, 6 were excluded because they were too frail for the home visit, 4 died, and 4 withdrew consent (Figure 2), with a dropout rate of $4.7 \%$. In the 85 participants that were measured at home, data collected were anthropometry, grip strength, and health questionnaires. Further follow-up measurements are being scheduled for 2017-2018.

\section{Measurements}

\section{Anthropometry and Questionnaires}

Sitting and standing height $(\mathrm{cm})$, weight $(\mathrm{kg})$, mid upper arm circumference, and four skinfold measurements were measured (triceps, biceps, subscapular, and suprailiac). Blood pressure was measured using the OMRON 705IT blood pressure monitor during sitting, supine, and standing, and systolic and diastolic readings were recorded. A musculoskeletal and general health questionnaire collected data on: activities of daily living (e.g., working 


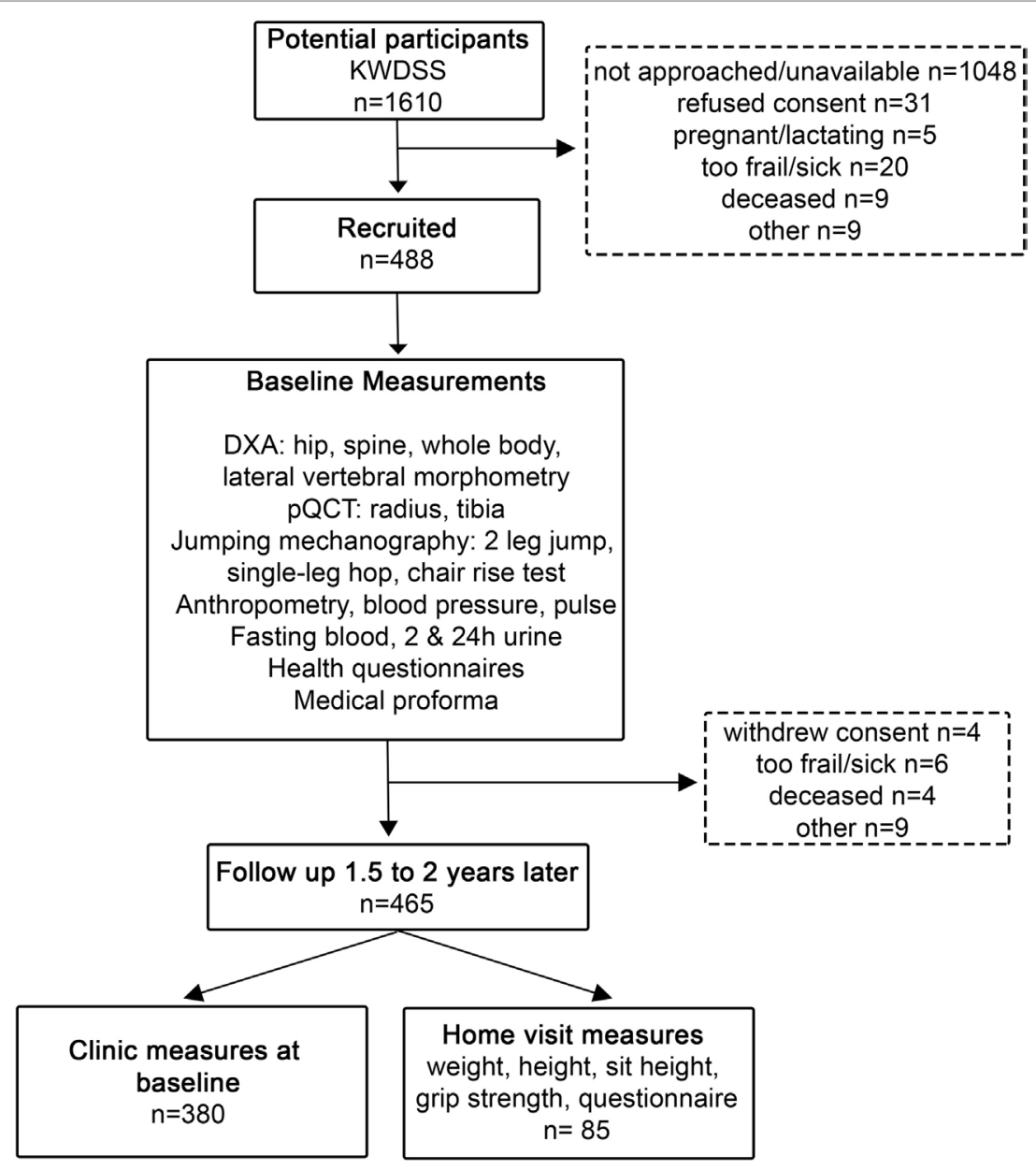

FIGURE 2 | Flow diagram of participant recruitment.

on a farm, gardening, performing five daily prayers, and fetching water), mental health, diagnosed illnesses (e.g., tuberculosis, diabetes mellitus, and leprosy), medication use, falls, fractures, musculoskeletal pain (located on specific body parts), lifestyle (occupational activities and domestic work), social demographics (including marital status, housing, and number of children), and medical history (through review of clinic medical records by the study physician/nurse). In women, there were also questions on menstrual cycle, breast-feeding history, pregnancy, parity, and menopausal status. Self-reported information on the use of medications and chronic medical conditions were collected.

\section{Blood and Urine Collection}

A $20 \mathrm{~mL}$ sample of venous blood was drawn from each participant in the early morning after an overnight fast; both lithium heparin and ethylene diamine tetra acetic acid (EDTA) plasma samples and an EDTA cell pellet for DNA extraction were stored at $-80^{\circ} \mathrm{C}$. Blood volume and all laboratory processing details were recorded on a data collection form. A 2-h fasting urine sample was collected, processed, and acidified and non-acidified samples were stored at $-20^{\circ} \mathrm{C}$. The 24 -h urine samples were stored at $-20^{\circ} \mathrm{C}$ in acidified and non-acidified aliquots; a further spun aliquot was stored at $-80^{\circ} \mathrm{C}$ for assessment of microalbuminuria. To date, markers of calcium, vitamin $\mathrm{D}$, bone metabolism, and kidney function have been measured.

\section{Dietary Assessment}

A 2-day prospective weighed dietary assessment was conducted in the participant's home at a time close to the measurement day by trained and experienced fieldworkers. This method has been used in previous studies of children, adults, and older people in Kiang West $(18,19)$. Briefly, fieldworkers visited participant's homes and recorded and weighed all food and drink items the participants consumed (total prepared minus amount left) over $48 \mathrm{~h}$. Data were coded and analysed using Diets-In, Nutrients-Out programme with Gambian food tables $(20,21)$.

\section{Dual-Energy X-ray Absorptiometry}

Each participant was scanned at the whole body, hip, spine, and forearm by DXA as previously described (22) (GE Lunar Prodigy, Waltham, MA, USA) software version 10; iDXA (GE Lunar 
Prodigy, Waltham, MA, USA) was introduced during the first follow-up and cross calibration data were obtained in 119 adults and children at the follow-up measurement. Bone outcomes were as follows: areal BMD (aBMD, $\left.\mathrm{g} / \mathrm{cm}^{2}\right), \mathrm{BMC}(\mathrm{g})$, and bone area $\left(\mathrm{BA}, \mathrm{cm}^{2}\right)$. Body composition outcomes were as follows: total body fat mass $(\mathrm{kg})$, total body lean mass $(\mathrm{kg})$, and appendicular lean mass $(\mathrm{kg})$. Fat and lean mass were also subdivided into the following regions: android, which is the lower abdominal area of the trunk between the ribs and the pelvis; gynoid, which is the region that encapsulates the hips, upper thighs, and buttocks. Lateral vertebral assessment (LVA) scans were obtained to determine whether there was any spinal degeneration, assessing osteoarthritic changes (osteophytes). Vertebral fractures were assessed semiautomatically in the GE Lunar software. All scans and fractures were assessed by a single reader, trained by an expert consultant musculoskeletal radiologist (Figures $3 \mathrm{~A}-\mathrm{C}$ ). Figure $3 \mathbf{A}$ is a representative LVA scan showing no signs of spinal degeneration compared to Figures 3B,C. Short-term precision, measured as coefficient of variation $(\mathrm{CV} \%)$ of duplicate measurements in 70 Gambian adults was $<1 \%$ for all sites for BMD.

\section{Peripheral Quantitative Computed Tomography}

Peripheral quantitative computed tomography measurements were made at the radius and tibia using a Stratec XCT-2000 scanner (Stratec, Pforzheim, Germany) software version 6.20c, as previously described (23). Measurements were taken at the following sites: 4,33 , and $66 \%$ radius; $4,14,38$, and $66 \%$ tibia. Forearm length was defined as the distance from the distal edge of the ulna styloid process to the olecranon. Leg length was defined as the distance from the most proximal edge of the medial malleolus to the intercondylar eminence. The scan sites were determined using a planar scout view of the distal radius or tibia and the reference line placed to bisect the lateral border of the end plate. The following outcome measures were taken at the $4 \%$ site: trabecular vBMD (Trab.vBMD, $\mathrm{mg} / \mathrm{cm}^{3}$ ), total vBMD (Total vBMD, $\mathrm{mg} / \mathrm{cm}^{3}$ ), cross-sectional area $\left(\mathrm{CSA}, \mathrm{mm}^{2}\right) ; 14,33,38$, and $66 \%$ site: CSA $\left(\mathrm{mm}^{2}\right)$, cortical vBMD (Ct.vBMD, $\left.\mathrm{mg} / \mathrm{cm}^{3}\right)$, cortical BMC (Ct. BMC, $\mathrm{mg} / \mathrm{mm})$, Ct.Area $\left(\mathrm{mm}^{2}\right)$, Ct.Thickness $(\mathrm{mm})$, medullary area $\left(\mathrm{mm}^{2}\right)$, stress strain index (SSI, $\left.\mathrm{mm}^{3}\right)$, cross-sectional moment of inertia $\left(\mathrm{CSMI}, \mathrm{mm}^{4}\right)$, periosteal circumference $(\mathrm{mm})$, endosteal circumference $(\mathrm{mm})$; and $66 \%$ site: cross-sectional muscle area $\left(\mathrm{CSMA}, \mathrm{mm}^{2}\right)$ and muscle density $\left(\mathrm{mg} / \mathrm{cm}^{3}\right)$. The range of CVs of duplicate measurements of bone outcomes in 62 Gambian adults was $1-4 \%$ for the radius and $1-3 \%$ for tibia measurements. The pQCT scans were also used to identify the presence or absence of vascular calcification at the $4,14,38$, and $66 \%$ sites of the tibia (Figures 3D-G).

\section{Jumping Mechanography}

To assess lower limb muscle function, a Leonardo Ground Reaction Force Platform (Leonardo software version 4.2; Novotec Medical GmbH, Pforzheim, Germany) was used as previously described (24-26). Participants were asked to perform three tests: a two-leg countermovement jump, a hopping test, and a chair rise test. Each measurement assesses different aspects of muscle strength, maximum power (jump), maximum force (hop), and time-to-stand (chair-rise). If a participant did not feel comfortable/confident in performing any or all of the jumping tests, they were not excluded from participating in other measurements.

\section{Grip Strength}

Grip strength was measured using a dynamometer (Jamar Hand Dynamometer, IL, USA) (27). The individual was seated in an upright position with the arm supported on the armrest of the chair with the wrist in a neutral position and the thumb facing upwards. Participants were instructed to exert maximal force. For each individual, we allowed one practice and then took three test measurements. The outcome measured was force $(\mathrm{kg})$.

\section{Ethics}

Ethics was obtained from the MRC Unit The Gambia Scientific Co-ordinating Committee (SCC) and joint Gambian Government/ MRC Unit The Gambia Ethics committee (SCC\#1222). All participants provided written informed consent. All procedures were carried out in accordance with the Declaration of Helsinki (28). If any participant had potential health problems identified by fieldworkers during recruitment or by the team during the field visit, they were advised to visit the clinic at MRC Keneba for follow-up and were offered transport. Participants with elevated blood pressure [according to WHO guidelines (29)] were followed up and treated as appropriate. Any other abnormalities were discussed with the study physician who followed-up appropriately. For instance, if musculoskeletal abnormalities were detected on the scan, the participant was referred for radiography at MRC Fajara.

\section{Power Calculation and Analyses}

Power calculations for GamBAS were determined to detect within individual change in femoral neck aBMD as it has the worst precision of all the sites measured with DXA. A sample size of 66 would be needed to detect a $1 \%$ change per annum over a random follow-up interval between 1.5 and 2 years later with a precision of $30 \%$ in the expected rate of change (described fully in Supplementary Material). To detect a 2\% change over the same time with $30 \%$ precision, we would need a sample size of 16 , or 37 for a precision of $20 \%$ in the expected rate of change. Thus, a minimum of 30 participants per 5 -year age band will be sufficient to identify rates of change within an individual of $1-2 \%$ per annum at the hip with confidence. In the other DXA and pQCT regions, which can be measured with more precision, smaller rates of change will be detectable with this number of participants. These rates are similar to or less than those observed in Caucasian populations during ageing and are biologically plausible and clinically relevant in terms of fracture risk $(30,31)$. Stratified sampling was used to ensure equal distribution of participants across each of the 5-year age bands, namely: 40-44.99, 45-49.99, 50-54.99, $55-59.99,60-64.99,65-69.99,70-74.99,75$ years and over; 239 men and 249 women were recruited. The scheduled follow-up measurements at a randomised interval between 1.5 and 2 years later for each participant will minimise the likelihood that the two measurements take place at exactly the same time of year. The exact interval for follow-up was different for each individual and assigned randomly at recruitment. This design was chosen since it allows us to account for inter-individual variation in patterns of 

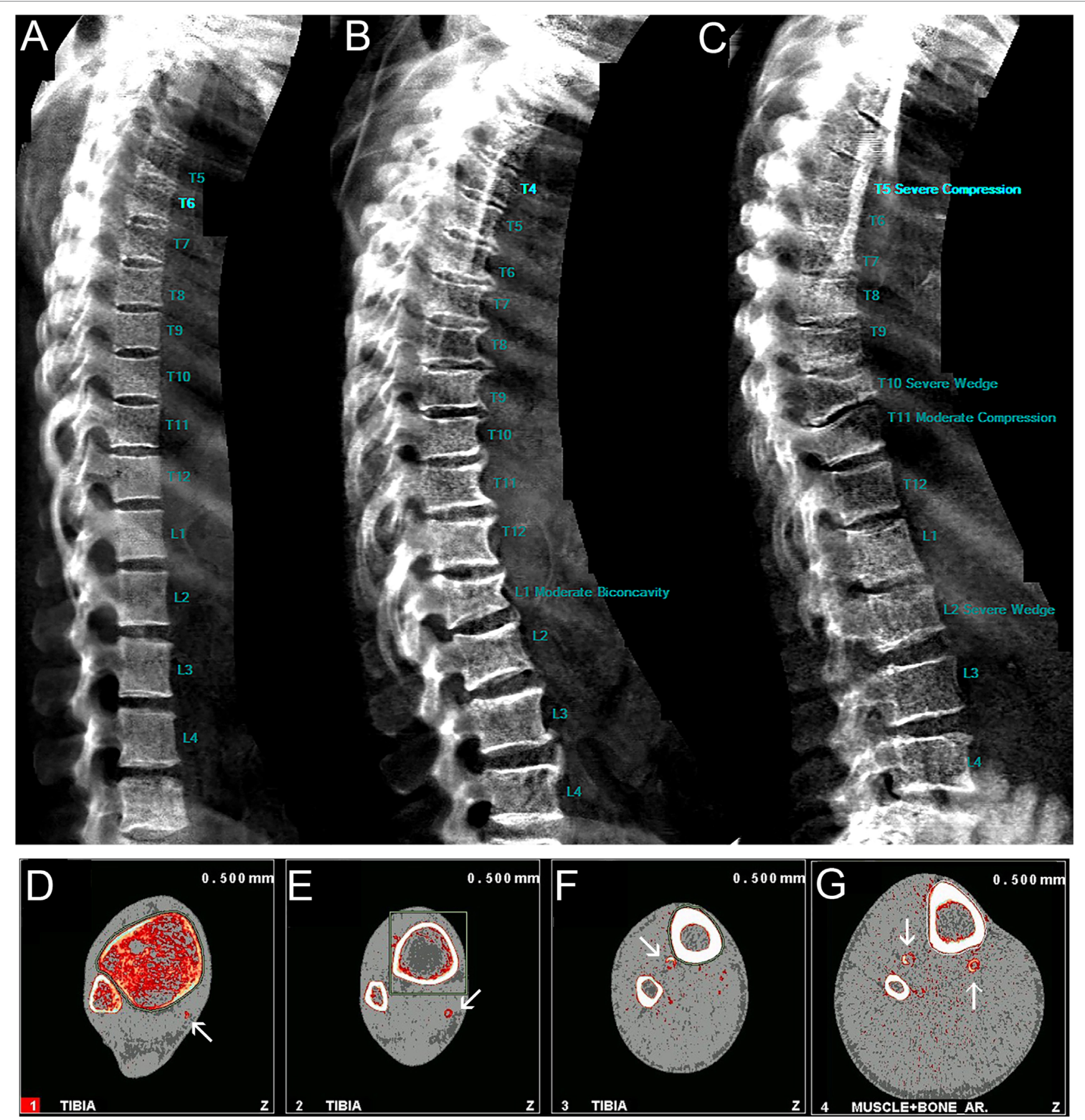

FIGURE 3 | Representative lateral vertebral assessment scans of (A) normal vertebrae, (B) biconcavity at the spine, (C) severe compression and wedge fractures; representative peripheral quantitative computed tomography scans with white arrows indicating vascular calcification from the (D) 4\%, (E) $14 \%$, (F) $38 \%$, and (G) $66 \%$ tibia.

seasonal change (Supplementary Material). Preliminary analyses on the baseline data include linear regression models to determine relationships between height, weight, musculoskeletal outcomes, and age. Analyses were split by sex due to patterns of age-related change in bone and muscle varying by sex. The data presented are from the baseline visits so all participants had measurements recorded for the primary and secondary outcomes. Some DXA and PQCT scans were excluded where significant motion artefact was detected which resulted in some missing data and were considered missing in these analyses. The missing data 
were independent of the participant's age and did not affect the preliminary analyses but may have reduced statistical power in some age bands. The participants who had missing data were not excluded from other measurements.

\section{RESULTS: KEY FINDINGS FROM BASELINE MEASUREMENTS}

\section{Population Characteristics}

Kiang West residents are mainly Muslim (3); GamBAS participants reported that they pray five times a day and most men and women were able to perform these prayers without physical difficulty (Table 1). Praying five times a day involves standing, kneeling, and rising from the floor multiple times. The questionnaire regarding musculoskeletal pain revealed that a higher proportion of women experienced musculoskeletal pain compared to men at the: back (51 vs $39 \%$ ), hip ( 49 vs $39 \%$ ), and knees (51 vs $40 \%$ ); this may potentially be due to the subsistence fieldwork being undertaken primarily by women (Table 1). The villages are divided into compounds where extended multigenerational families live together. Polygamy is practiced in this population; women have one husband at any one time whereas

TABLE 1 | Lifestyle outcomes in men and women.

\begin{tabular}{|c|c|c|}
\hline & Men $(n=239)$ & Women $(n=249)$ \\
\hline \multicolumn{3}{|l|}{ Number of marriages } \\
\hline Never married & $6(3)$ & $8(3)$ \\
\hline Once & $217(91)$ & $120(48)$ \\
\hline Twice & $8(3)$ & $65(26)$ \\
\hline Three or more & $2(1)$ & $56(22)$ \\
\hline Missing data & $6(3)$ & - \\
\hline \multicolumn{3}{|l|}{ Living arrangement } \\
\hline Alone & $5(2)$ & $16(6)$ \\
\hline With 1 spouse and no children & $4(2)$ & $3(1)$ \\
\hline With 1 spouse and children & $128(54)$ & - \\
\hline With co-wives/spouses & $3(1)$ & $134(54)$ \\
\hline With children only & $2(1)$ & $83(33)$ \\
\hline With co-wives/spouses and children & $89(37)$ & - \\
\hline Others & - & $12(5)$ \\
\hline Missing data & $8(3)$ & $1(0)$ \\
\hline \multicolumn{3}{|l|}{ Smoking status } \\
\hline Non-smoker & $181(76)$ & $202(81)$ \\
\hline Current smoker & $55(23)$ & $39(16)$ \\
\hline Missing data & $3(1)$ & $8(3)$ \\
\hline \multicolumn{3}{|l|}{ Farm or field work } \\
\hline No & $36(15)$ & $25(10)$ \\
\hline Used to but not anymore & $8(3)$ & $7(3)$ \\
\hline Yes currently & $192(80)$ & $211(85)$ \\
\hline Missing data & $3(1)$ & $6(2)$ \\
\hline \multicolumn{3}{|l|}{ Perform five daily prayers } \\
\hline Yes, with difficulty but without help & $15(6)$ & $8(3)$ \\
\hline Yes, without difficulty & $220(92)$ & $232(93)$ \\
\hline Only with help & - & $1(0)$ \\
\hline Missing data & $4(2)$ & $8(3)$ \\
\hline \multicolumn{3}{|l|}{ Musculoskeletal pain } \\
\hline Back pain & $94(39)$ & $128(51)$ \\
\hline Hip pain & $92(39)$ & $121(49)$ \\
\hline Knee pain & $95(40)$ & $126(51)$ \\
\hline
\end{tabular}

Values are frequency (\%). men may have up to four wives. Women often live in one hut with their co-wives and men often live separately; $99.5 \%$ of men and $98.8 \%$ of women reside in dwellings with corrugate roofs. Women had a median of 9 (IQR: 7-10) children that were born alive. The main livelihood is rural subsistence farming where income and eating patterns depend on available foods that fluctuate throughout the year and are greatly influenced by the annual rainy season (June to October). This influences intakes of specific foods, nutrients, and energy intake. Dietary intakes of key micronutrients remain low and relatively unchanged to our previous work. Overall, men had higher intakes of all micronutrients. Some notable sex differences include a $21 \%$ greater daily habitual calcium intake in men than in women (Table 2). The greatest sex difference was seen in daily habitual iron intake, where men had a $33 \%$ greater daily iron intake compared to women. Across the age bands, daily habitual calcium intake [mean (SD)] was 378.0 (176.0) $\mathrm{mg} /$ day in men and 295.9 (175.9) $\mathrm{mg} /$ day in women (Table 2).

\section{Bone, Body Composition, and Age}

Baseline data describing anthropometry and body composition in men and women are shown in Tables 3 and 4 . In men, the most significant negative associations with age were for weight $(-0.29 \mathrm{~kg}, p<0.0001)$ and total body lean mass $(-0.24$, $p<0.0001)$. In women, anthropometry and body composition outcomes all had significant negative associations with age, with the greatest difference observed in weight $(-0.26 \mathrm{~kg}, p<0.0001)$. Men had greater negative differences in total body lean mass with age than women following adjustments for weight and height $\left(R^{2}=0.90\right.$, interaction $\left.p<0.0001\right)$. Waist circumference and body fat outcomes were not different with age in both men and women.

Dual energy X-ray absorptiometry bone parameters in men and women are displayed in Tables 5 and $\mathbf{6}$, respectively. Baseline cross-sectional data show preliminary evidence for bone and muscle loss with age. In both men and women, there were negative associations between age, and DXA bone outcomes at all sites, particularly clinically relevant sites including spine, total hip, and femoral neck aBMD (Figures $4 \mathrm{~A}-\mathrm{C}$ ). There were positive associations with BA and age at the total hip and radius in both men and women following adjustments (Tables 5 and 6).

TABLE 2 | Nutritional intake of men and women.

\begin{tabular}{lccc}
\hline & Men $(\boldsymbol{n}=\mathbf{2 2 5})^{\mathbf{a}}$ & Women $(\boldsymbol{n}=\mathbf{2 4 2})^{\mathbf{a}}$ & $\boldsymbol{p}$-value \\
\hline Calcium (mg/day) & $378.0 \pm 176.0$ & $295.9 \pm 175.9$ & $<\mathbf{0 . 0 0 0 1}$ \\
Phosphorus (mg/day) & $836.4 \pm 275.4$ & $620.2 \pm 243.4$ & $<\mathbf{0 . 0 0 0 1}$ \\
Iron (mg/day) & $37.2 \pm 25.8$ & $25.0 \pm 16.5$ & $<\mathbf{0 . 0 0 0 1}$ \\
Zinc (mg/day) & $9.3 \pm 3.0$ & $7.0 \pm 2.8$ & $<\mathbf{0 . 0 0 0 1}$ \\
Dietary fibres (mg/day) & $44.4 \pm 14.2$ & $33.9 \pm 12.4$ & $<\mathbf{0 . 0 0 0 1}$ \\
Phytate (g/day) & $1.3 \pm 0.5$ & $1.0 \pm 0.4$ & $<\mathbf{0 . 0 0 0 1}$ \\
Potassium (mg/day) & $2,409.0 \pm 868.9$ & $1,800.1 \pm 705.4$ & $<\mathbf{0 . 0 0 0 1}$ \\
Magnesium (mg/day) & $527.3 \pm 192.9$ & $388.4 \pm 150.4$ & $<\mathbf{0 . 0 0 0 1}$
\end{tabular}

Values are mean $\pm S D$.

Bold indicates significance.

Dietary intakes were estimated from 2-day weighed diet diaries, and intakes calculated from Gambian food tables.

${ }^{2} 21$ participants did not have dietary information available. 
TABLE 3 | Anthropometry and body composition in men.

\begin{tabular}{|c|c|c|c|c|c|c|c|c|c|c|}
\hline Age (yr) & 40-44 $(n=25)$ & $45-49(n=34)$ & $\mathbf{5 0 - 5 4}(n=29)$ & 55-59 $(n=31)$ & $60-64(n=27)$ & $65-69(n=35)$ & 70-74 $(n=30)$ & $75+(n=28)$ & $\beta$-Coefficient (95\% Cl) & $p$-value \\
\hline Weight (kg) & $66.9 \pm 10.8$ & $61.5 \pm 9.5$ & $64.1 \pm 12.3$ & $59.1 \pm 10.8$ & $59.2 \pm 8.4$ & $59.5 \pm 9.6$ & $55.8 \pm 7.6$ & $53.8 \pm 7.5$ & $-0.29(-0.39,-0.19)$ & $<0.0001$ \\
\hline Height (cm) & $171.2 \pm 5.1$ & $171.0 \pm 5.9$ & $171.2 \pm 6.7$ & $170.3 \pm 6.8$ & $169.1 \pm 8.7$ & $167.4 \pm 7.0$ & $168.7 \pm 7.9$ & $164.9 \pm 6.0$ & $-0.16(-0.23,-0.08)$ & $<0.0001$ \\
\hline Sitting height (cm) & $86.2 \pm 3.7$ & $85.2 \pm 3.1$ & $85.1 \pm 4.1$ & $84.0 \pm 3.4$ & $83.7 \pm 4.0$ & $82.9 \pm 3.6$ & $83.1 \pm 4.2$ & $81.2 \pm 2.7$ & $-0.11(-0.15,-0.08)$ & $<0.0001$ \\
\hline Sit:stand height ratio & $0.50 \pm 0.02$ & $0.50 \pm 0.01$ & $0.50 \pm 0.01$ & $0.49 \pm 0.01$ & $0.50 \pm 0.01$ & $0.50 \pm 0.01$ & $0.49 \pm 0.01$ & $0.49 \pm 0.02$ & $-0.0002(-0.0004,-0.00008)$ & 0.003 \\
\hline BMl & $22.8 \pm 3.7$ & $21.0 \pm 3.0$ & $21.8 \pm 3.5$ & $20.3 \pm 2.9$ & $20.7 \pm 2.8$ & $21.2 \pm 3.2$ & $19.6 \pm 2.3$ & $19.8 \pm 2.5$ & $-0.06(-0.09,-0.03)$ & $<0.0001$ \\
\hline Waist circumference $(\mathrm{cm})$ & $79.2 \pm 8.5$ & $76.5 \pm 8.0^{(n=32)}$ & $80.1 \pm 8.4$ & $77.8 \pm 8.5$ & $77.1 \pm 17.1$ & $79.2 \pm 9.3$ & $76.1 \pm 7.9^{(n=29)}$ & $75.8 \pm 8.6$ & $-0.06(-0.16,0.04)$ & 0.243 \\
\hline Total body fat mass (kg) & $11.0 \pm 6.6^{(n=24)}$ & $7.2 \pm 5.3$ & $9.7 \pm 7.5$ & $7.2 \pm 6.0$ & $8.1 \pm 5.4$ & $9.3 \pm 6.7$ & $7.3 \pm 4.6^{(n=29)}$ & $7.1 \pm 4.2^{(n=26)}$ & $-0.05(-0.12,0.01)$ & 0.120 \\
\hline Total\% fat & $15.5 \pm 7.3^{(n=24)}$ & $11.0 \pm 6.4$ & $14.1 \pm 8.4$ & $11.1 \pm 6.9$ & $13.0 \pm 7.3$ & $14.6 \pm 8.1$ & $12.6 \pm 6.6^{(n=29)}$ & $12.7 \pm 6.2^{(n=26)}$ & $-0.007(-0.08,0.08)$ & 0.865 \\
\hline Android fat mass (kg) & $0.9 \pm 0.7$ & $0.6 \pm 0.5$ & $0.8 \pm 0.7$ & $0.6 \pm 0.6$ & $0.7 \pm 0.6$ & $0.8 \pm 0.7$ & $0.5 \pm 0.4$ & $0.6 \pm 0.5^{(n=26)}$ & $-0.004(-0.01,0.002)$ & 0.203 \\
\hline Gynoid fat mass (kg) & $2.2 \pm 1.1^{(n=24)}$ & $1.5 \pm 1.0$ & $1.9 \pm 1.3$ & $1.4 \pm 1.0$ & $1.6 \pm 0.9$ & $1.8 \pm 1.0$ & $1.5 \pm 0.9^{(n=29)}$ & $1.4 \pm 0.7^{(n=27)}$ & $-0.01(-0.02,0.0006)$ & 0.051 \\
\hline FMl $\left(\mathrm{kg} / \mathrm{m}^{2}\right)$ & $3.8 \pm 2.3^{(n=24)}$ & $2.5 \pm 1.7$ & $3.3 \pm 2.5$ & $2.4 \pm 1.9$ & $2.8 \pm 2.0$ & $3.3 \pm 2.4$ & $2.6 \pm 1.6^{(n=29)}$ & $2.6 \pm 1.6^{(n=26)}$ & $-0.01(-0.03,0.01)$ & 0.292 \\
\hline Total body lean mass $(\mathrm{kg})$ & $53.2 \pm 5.6^{(n=24)}$ & $51.7 \pm 5.8$ & $51.5 \pm 6.9$ & $49.5 \pm 5.9$ & $48.4 \pm 5.1$ & $47.6 \pm 4.9$ & $45.8 \pm 5.5^{(n=29)}$ & $44.1 \pm 5.0^{(n=26)}$ & $-0.24(-0.29,-0.18)$ & $<0.0001$ \\
\hline aLM (kg) & $25.6 \pm 3.1$ & $24.6 \pm 3.5$ & $24.6 \pm 3.9$ & $23.2 \pm 3.2$ & $22.4 \pm 2.6$ & $21.9 \pm 2.8$ & $20.9 \pm 3.0$ & $20.0 \pm 2.9^{(n=27)}$ & $-0.1(-0.2,-0.1)$ & $<0.0001$ \\
\hline Android lean mass (kg) & $3.4 \pm 0.5$ & $3.3 \pm 0.4$ & $3.3 \pm 0.5$ & $3.2 \pm 0.4$ & $3.3 \pm 0.4$ & $3.2 \pm 0.4$ & $3.2 \pm 0.5$ & $2.9 \pm 0.4^{(n=26)}$ & $-0.009(-0.01,-0.004)$ & $<0.0001$ \\
\hline Gynoid lean mass (kg) & $7.7 \pm 1.1^{(n=24)}$ & $7.4 \pm 1.1$ & $7.3 \pm 1.2$ & $6.9 \pm 0.9$ & $6.8 \pm 0.9$ & $6.7 \pm 0.8$ & $6.5 \pm 0.8^{(n=29)}$ & $6.2 \pm 0.7^{(n=27)}$ & $-0.04(-0.05,-0.03)$ & $<0.0001$ \\
\hline aLMI $\left(\mathrm{kg} / \mathrm{m}^{2}\right)$ & $8.7 \pm 0.9$ & $8.4 \pm 1.1$ & $8.4 \pm 1.0$ & $8.0 \pm 0.8$ & $7.8 \pm 0.7$ & $7.8 \pm 0.8$ & $7.3 \pm 0.8$ & $7.3 \pm 0.8^{(n=27)}$ & $-0.04(-0.05,-0.03)$ & $<0.0001$ \\
\hline
\end{tabular}

Values are mean $\pm S D$.

$\beta$-Coefficients are calculated with age as a continuous variable.

Superscript values indicate the group numbers.

Bold indicates significance.

BMI, body mass index; FMI, fat mass index, calculated as whole body fat mass divided by height squared; aLM, appendicular lean mass; aLMI, appendicular lean mass index, calculated as appendicular lean mass divided by height squared.

TABLE 4 | Anthropometry and body composition in women.

\begin{tabular}{|c|c|c|c|c|c|c|c|c|c|c|}
\hline & $40-44(n=28)$ & $45-49(n=32)$ & $50-54(n=30)$ & $55-59(n=31)$ & $60-64(n=31)$ & $65-69(n=33)$ & $70-74(n=30)$ & $75+(n=34)$ & $\beta$-Coefficient $(95 \% \mathrm{Cl})$ & $p$-value \\
\hline Weight (kg) & $58.1 \pm 11.5$ & $60.8 \pm 11.4$ & $57.1 \pm 10.8$ & $53.8 \pm 9.6$ & $53.4 \pm 7.2$ & $53.5 \pm 9.6$ & $52.2 \pm 9.9$ & $49.3 \pm 8.5$ & $-0.26(-0.35,-0.16)$ & $<0.0001$ \\
\hline Height (cm) & $59.3 \pm 5.1$ & $159.8 \pm 6.1$ & $158.6 \pm 6.2$ & $158.1 \pm 5.8$ & $157.6 \pm 4.9$ & $160.1 \pm 5.7$ & $154.8 \pm 5.7$ & $154.0 \pm 5.7$ & $-0.14(-0.20,-0.09)$ & $<0.0001$ \\
\hline Sitting height (cm) & $81.7 \pm 2.8$ & $1.2 \pm 3.5$ & $80.4 \pm 2.9$ & $79.1 \pm 3.8$ & $79.5 \pm 3.1$ & $80.2 \pm 3.5$ & $77.8 \pm 3.3$ & $76.5 \pm 3.3$ & $-0.13(-0.16,-0.09)$ & $<0.0001$ \\
\hline Sit:stand height ratio & $0.51 \pm 0.02$ & $0.51 \pm 0.01$ & $0.51 \pm 0.02$ & $0.50 \pm 0.02$ & $0.50 \pm 0.01$ & $0.50 \pm 0.02$ & $0.50 \pm 0.02$ & $0.50 \pm 0.01$ & $-0.0004(-0.0005,-0.0002)$ & $<0.0001$ \\
\hline BMI & $22.9 \pm 4.4$ & $23.9 \pm 4$ & $22.7 \pm 4.3$ & $21.4 \pm 3.1$ & $21.4 \pm 2.3$ & $20.8 \pm 3.2$ & $21.7 \pm 3.7$ & $20.7 \pm 2.8$ & $-0.07(-0.10,-0.03)$ & $<0.0001$ \\
\hline Waist circumference $(\mathrm{cm})$ & $70.7 \pm 10.1$ & $75.7 \pm 9.7$ & $72.0 \pm 8.6$ & $70.6 \pm 6.6$ & $71.4 \pm 6.3^{(n=29)}$ & $71.0 \pm 7.1^{(n=29)}$ & $73.3 \pm 8.5^{(n=23)}$ & $68.4 \pm 5.4^{(n=19)}$ & $-0.06(-0.14,0.03)$ & 0.203 \\
\hline Total body fat mass (kg) & $18.4 \pm 8.7^{(n=27)}$ & $20.7 \pm 9.3$ & $18.3 \pm 8.3$ & $16.3 \pm 6.7^{(n=30)}$ & $16.0 \pm 4.8$ & $16.1 \pm 6.8$ & $16.4 \pm 6.7^{(n=29)}$ & $14.1 \pm 5.5^{(n=30)}$ & $-0.12(-0.20,-0.05)$ & 0.001 \\
\hline Total\% fat & $30.3 \pm 8.1^{(n=27)}$ & $32.5 \pm 10.0$ & $30.8 \pm 8.7$ & $29.3 \pm 8.2^{(n=30)}$ & $29.6 \pm 6.0$ & $29.0 \pm 7.6$ & $30.4 \pm 7.9^{(n=29)}$ & $27.9 \pm 7.1^{(n=30)}$ & $-0.07(-0.15,0.01)$ & 0.09 \\
\hline Android fat mass (kg) & $0.9^{(n=27)}$ & $1.3 \pm 0.8$ & & $0.9 \pm 0.5^{(n=30)}$ & & & $1.0 \pm 0.6^{(n=29)}$ & $0.8 \pm 0.5^{(n=31)}$ & $1,-0.001)$ & 0.02 \\
\hline mass $(\mathrm{kg})$ & $4.1 \pm 1.5^{(n=27)}$ & $4.3 \pm 1.6$ & $9 \pm 1.3$ & $3.4 \pm 1.2$ & $3.5 \pm 1.0$ & $3.4 \pm 1.2$ & $3.2 \pm 1.1$ & $2.9 \pm 1.0^{(n=33)}$ & $-0.03(-0.04,-0.02)$ & $<0.0001$ \\
\hline FMI $\left(\mathrm{kg} / \mathrm{m}^{2}\right)$ & $7.2 \pm 3.4^{(n=27)}$ & $8.1 \pm 3.7$ & $7.3 \pm 3.4$ & $6.5 \pm 2.6^{(n=30)}$ & $6.4 \pm 1.8$ & $6.2 \pm 2.5$ & $6.9 \pm 2.7^{(n=29)}$ & $6.0 \pm 2.2^{(n=30)}$ & $-0.04(-0.07,-0.01)$ & 0.009 \\
\hline Total body lean mass (kg) & $36.7 \pm 4.1^{(n=27)}$ & $37.0 \pm 4.4$ & $35.7 \pm 4.0$ & $35.0 \pm 4.5^{(n=30)}$ & $34.7 \pm 3.6$ & $34.7 \pm 3.4$ & $33.4 \pm 4.7^{(n=29)}$ & $32.5 \pm 4.3^{(n=30)}$ & $-0.11(-0.16,-0.07)$ & $<0.0001$ \\
\hline aLM $(\mathrm{kg})$ & $16.9 \pm 2.3^{(n=27)}$ & $16.9 \pm 2.2$ & $16.1 \pm 2.3$ & $15.6 \pm 2.3$ & $15.4 \pm 2.1$ & $15.3 \pm 2.0$ & $14.7 \pm 2.4$ & $14.1 \pm 2.2$ & $-0.07(-0.10,-0.05)$ & $<0.0001$ \\
\hline Android lean mass (kg) & $2.3 \pm 0.3^{(n=27)}$ & $2.4 \pm 0.4$ & $2.3 \pm 0.3$ & $2.2 \pm 0.3^{(n=30)}$ & $2.2 \pm 0.2$ & $2.2 \pm 0.2$ & $2.2 \pm 0.4^{(n=29)}$ & $2.2 \pm 0.3^{(n=31)}$ & $-0.006(-0.009,-0.003)$ & 0.001 \\
\hline Gynoid lean mass (kg) & $5.2 \pm 0.9^{(n=27)}$ & $5.2 \pm 0.7$ & $5.0 \pm 0.6$ & $4.7 \pm 0.8$ & $4.8 \pm 0.7$ & $4.8 \pm 0.7$ & $4.5 \pm 0.7$ & $4.4 \pm 0.6^{(n=33)}$ & $-0.02(-0.03,-0.01)$ & $<0.0001$ \\
\hline $\operatorname{aLMl}\left(\mathrm{kg} / \mathrm{m}^{2}\right)$ & $6.6 \pm 0.8^{(n=27)}$ & $6.6 \pm 0.8$ & $6.4 \pm 0.7$ & $6.2 \pm 0.6$ & $6.2 \pm 0.7$ & $5.9 \pm 0.6$ & $6.1 \pm 0.8$ & $5.9 \pm 0.7$ & $-0.02(-0.03,-0.01)$ & $<0.0001$ \\
\hline
\end{tabular}

Values are mean $\pm S D$.

$\beta$-Coefficients are calculated with age as a continuous variable.

Superscript values indicate the group numbers.

Bold indicates significance.

BMI, body mass index; FMI, fat mass index, calculated as whole body fat mass divided by height squared; aLM, appendicular lean mass; aLMI, appendicular lean mass index, calculated as appendicular lean mass divided by height

squared. 
TABLE 5 | Dual energy X-ray absorptiometry bone parameters in men.

\begin{tabular}{|c|c|c|c|c|c|c|c|c|c|c|c|c|c|c|}
\hline Age (yr) & $\begin{array}{l}\mathbf{4 0 - 4 4} \\
(n=25)\end{array}$ & $\begin{array}{l}\mathbf{4 5 - 4 9} \\
(n=34)\end{array}$ & $\begin{array}{l}\mathbf{5 0 - 5 4} \\
(n=29)\end{array}$ & $\begin{array}{l}\mathbf{5 5 - 5 9} \\
(n=31)\end{array}$ & $\begin{array}{l}60-64 \\
(n=27)\end{array}$ & $\begin{array}{l}65-69 \\
(n=35)\end{array}$ & $\begin{array}{c}70-74 \\
(n=30)\end{array}$ & $\begin{array}{c}75+ \\
(n=28)\end{array}$ & $\begin{array}{c}\beta \text {-Coefficient } \\
(95 \% \mathrm{Cl})\end{array}$ & $R^{2}$ & $\begin{array}{l}\text { Unadjusted } \\
p \text {-value }\end{array}$ & $\begin{array}{c}\beta \text {-Coefficient } \\
(95 \% \mathrm{Cl})\end{array}$ & $R^{2}$ & $\begin{array}{c}\text { Adjusted } \\
p \text {-value }\end{array}$ \\
\hline \multicolumn{15}{|c|}{ Whole body } \\
\hline $\begin{array}{l}\mathrm{aBMD} \\
\left(\mathrm{g} / \mathrm{cm}^{2}\right)\end{array}$ & $1.2 \pm 0.1^{(n=24)}$ & $1.2 \pm 0.1$ & $1.2 \pm 0.1$ & $1.1 \pm 0.1$ & $1.1 \pm 0.1$ & $1.1 \pm 0.1$ & $1.1 \pm 0.1^{(n=29)}$ & $1.1 \pm 0.1^{(n=26)}$ & $\begin{array}{l}-0.003 \\
(-0.004 \\
-0.002)\end{array}$ & 0.13 & $<0.0001$ & $\begin{array}{c}-0.002 \\
(-0.003 \\
-0.0008)\end{array}$ & 0.34 & $<0.0001$ \\
\hline $\begin{array}{l}\text { BMC } \\
\text { (g) }\end{array}$ & $2,804 \pm 433^{(n=24)}$ & $2,632 \pm 358$ & $2,739 \pm 433$ & $2,532 \pm 374$ & $2,442 \pm 448$ & $2,433 \pm 366$ & $2,335 \pm 299^{(n=29)}$ & $2,279 \pm 380^{(n=26)}$ & $\begin{array}{c}-13.3(-17.4 \\
-9.2)\end{array}$ & 0.15 & $<0.0001$ & $\begin{array}{c}-3.9(-6.6 \\
-1.1)\end{array}$ & 0.66 & 0.007 \\
\hline $\begin{array}{l}\mathrm{BA} \\
\left(\mathrm{cm}^{2}\right)\end{array}$ & $2,355 \pm 200^{(n=24)}$ & $2,275 \pm 212$ & $2,321 \pm 233$ & $2,242 \pm 217$ & $2,210 \pm 243$ & $2,175 \pm 215$ & $2,171 \pm 204^{(n=29)}$ & $2,087 \pm 205^{(n=26)}$ & $\begin{array}{c}-6.2(-8.5 \\
-3.9)\end{array}$ & 0.11 & $<0.0001$ & $\begin{array}{c}-0.1(-1.2 \\
1.0)\end{array}$ & 0.83 & 0.856 \\
\hline \multicolumn{15}{|l|}{ Spine } \\
\hline $\begin{array}{l}\text { aBMD } \\
\left(\mathrm{g} / \mathrm{cm}^{2}\right)\end{array}$ & $1.1 \pm 0.2^{(n=24)}$ & $1.0 \pm 0.1$ & $1.1 \pm 0.1$ & $1.0 \pm 0.1^{(n=30)}$ & $1.0 \pm 0.2$ & $1.0 \pm 0.2$ & $1.0 \pm 0.2$ & $1.0 \pm 0.2^{(n=26)}$ & $\begin{array}{l}-0.002 \\
(-0.004 \\
-0.0004)\end{array}$ & 0.03 & 0.014 & $\begin{array}{c}-0.0006 \\
(-0.002 \\
0.001)\end{array}$ & 0.13 & 0.486 \\
\hline $\begin{array}{l}\text { BMC } \\
\text { (g) }\end{array}$ & $61.1 \pm 12.6^{(n=24)}$ & $57.1 \pm 8.9$ & $59.6 \pm 11.0$ & $54.5 \pm 9.3^{(n=30)}$ & $55.9 \pm 17.6$ & $55.4 \pm 11.3$ & $54.2 \pm 10.8$ & $53.5 \pm 13.5^{(n=26)}$ & $\begin{array}{c}-0.1(-0.3 \\
-0.02)\end{array}$ & 0.02 & 0.025 & $\begin{array}{c}0.06(-0.05 \\
0.17)\end{array}$ & 0.31 & 0.306 \\
\hline $\begin{array}{l}\mathrm{BA} \\
\left(\mathrm{cm}^{2}\right)\end{array}$ & $55.7 \pm 5.2^{(n=24)}$ & $55.7 \pm 4.0$ & $56.4 \pm 5.8$ & $54.8 \pm 5.2^{(n=30)}$ & $55.9 \pm 7.2$ & $54.9 \pm 4.8$ & $55.6 \pm 6.8$ & $54.6 \pm 5.6^{(n=26)}$ & $\begin{array}{c}-0.02(-0.08 \\
0.03)\end{array}$ & 0.003 & 0.405 & $\begin{array}{c}0.09(0.05 \\
0.13)\end{array}$ & 0.56 & $<0.0001$ \\
\hline \multicolumn{15}{|c|}{ Total hip } \\
\hline $\begin{array}{l}\mathrm{aBMD} \\
\left(\mathrm{g} / \mathrm{cm}^{2}\right)\end{array}$ & $1.1 \pm 0.2$ & $1.0 \pm 0.1$ & $1.1 \pm 0.1$ & $1.0 \pm 0.1^{(n=29)}$ & $0.9 \pm 0.2$ & $1.0 \pm 0.1^{(n=33)}$ & $0.9 \pm 0.1^{(n=29)}$ & $0.9 \pm 0.2^{(n=27)}$ & $\begin{array}{l}-0.004 \\
(-0.006 \\
-0.003)\end{array}$ & 0.14 & $<0.0001$ & $\begin{array}{l}-0.003 \\
(-0.005 \\
-0.002)\end{array}$ & 0.20 & $<0.0001$ \\
\hline $\begin{array}{l}\mathrm{BMC} \\
\text { (g) }\end{array}$ & $35.4 \pm 6.2$ & $34.2 \pm 4.2$ & $35.8 \pm 4.5$ & $33.0 \pm 4.4^{(n=29)}$ & $31.4 \pm 5.9$ & $31.6 \pm 4.7^{(n=33)}$ & $31.1 \pm 4.4^{(n=29)}$ & $29.7 \pm 6.3^{(n=27)}$ & $\begin{array}{c}-0.15(-0.2 \\
-0.1)\end{array}$ & 0.12 & $<0.0001$ & $\begin{array}{c}-0.07(-0.1 \\
-0.02)\end{array}$ & 0.32 & 0.005 \\
\hline $\begin{array}{l}\mathrm{BA} \\
\left(\mathrm{cm}^{2}\right)\end{array}$ & $33.3 \pm 2.1$ & $33.5 \pm 2.6$ & $34.1 \pm 2.3$ & $33.4 \pm 2.2^{(n=29)}$ & $33.8 \pm 2.6$ & $33.0 \pm 2.1^{(n=33)}$ & $34.0 \pm 2.0^{(n=29)}$ & 33. $0 \pm 2.2^{(n=27)}$ & $\begin{array}{c}-0.009(-0.03 \\
0.02)\end{array}$ & 0.002 & 0.468 & $\begin{array}{c}0.04(0.02 \\
0.06)\end{array}$ & 0.52 & $<0.0001$ \\
\hline \multicolumn{15}{|c|}{ Femoral neck } \\
\hline $\begin{array}{l}\mathrm{aBMD} \\
\left(\mathrm{g} / \mathrm{cm}^{2}\right)\end{array}$ & $1.0 \pm 0.1$ & $1.0 \pm 0.1$ & $1.0 \pm 0.1$ & $0.9 \pm 0.1^{(n=30)}$ & $0.9 \pm 0.1$ & $0.9 \pm 0.1$ & $0.8 \pm 0.1^{(n=29)}$ & $0.8 \pm 0.2^{(n=27)}$ & $\begin{array}{l}-0.005 \\
(-0.007 \\
-0.004)\end{array}$ & 0.22 & $<0.0001$ & $\begin{array}{l}-0.004 \\
(-0.006 \\
-0.003)\end{array}$ & 0.31 & $<0.0001$ \\
\hline $\begin{array}{l}\text { BMC } \\
(g)\end{array}$ & $5.0 \pm 1.0$ & $4.7 \pm 0.7$ & $5.0 \pm 0.7$ & $4.4 \pm 0.6^{(n=30)}$ & $4.3 \pm 0.8$ & $4.3 \pm 0.7$ & $4.0 \pm 0.6^{(n=29)}$ & $3.8 \pm 0.8^{(n=27)}$ & $\begin{array}{c}-0.03(-0.04 \\
-0.02)\end{array}$ & 0.20 & $<0.0001$ & $\begin{array}{c}-0.02(-0.03 \\
-0.01)\end{array}$ & 0.36 & $<0.0001$ \\
\hline $\begin{array}{l}\mathrm{BA} \\
\left(\mathrm{cm}^{2}\right)\end{array}$ & $4.8 \pm 0.6$ & $5.0 \pm 0.6$ & $5.1 \pm 0.5$ & $4.8 \pm 0.5^{(n=30)}$ & $5.0 \pm 0.5$ & $5.0 \pm 0.5$ & $4.8 \pm 0.4^{(n=29)}$ & $4.8 \pm 0.6^{(n=27)}$ & $\begin{array}{c}-0.003 \\
(-0.009,0.002)\end{array}$ & 0.01 & 0.264 & $\begin{array}{c}0.002(-0.003 \\
0.008)\end{array}$ & 0.13 & 0.382 \\
\hline \multicolumn{15}{|l|}{ Radius } \\
\hline $\begin{array}{l}\text { aBMD } \\
\left(\mathrm{g} / \mathrm{cm}^{2}\right)\end{array}$ & $0.7 \pm 0.1$ & $0.7 \pm 0.1$ & $0.7 \pm 0.1$ & $0.7 \pm 0.1$ & $0.7 \pm 0.1$ & $0.7 \pm 0.1$ & $0.6 \pm 0.1$ & $0.6 \pm 0.1^{(n=27)}$ & $\begin{array}{l}-0.004 \\
(-0.005 \\
-0.003)\end{array}$ & 0.25 & $<0.0001$ & $\begin{array}{l}-0.003 \\
(-0.004 \\
-0.002)\end{array}$ & 0.34 & $<0.0001$ \\
\hline $\begin{array}{l}\mathrm{BMC} \\
(\mathrm{g})\end{array}$ & $12.9 \pm 1.6$ & $12.2 \pm 1.7$ & $12.5 \pm 1.9$ & $12.0 \pm 1.8$ & $11.4 \pm 1.8$ & $11.3 \pm 2.1$ & $10.6 \pm 1.5$ & $10.2 \pm 2.0^{(n=27)}$ & $\begin{array}{c}-0.07(-0.09 \\
-0.05)\end{array}$ & 0.17 & $<0.0001$ & $\begin{array}{c}-0.03(-0.05 \\
-0.02)\end{array}$ & 0.49 & $<0.0001$ \\
\hline $\begin{array}{l}\mathrm{BA} \\
\left(\mathrm{cm}^{2}\right)\end{array}$ & $17.3 \pm 1.6$ & $17.1 \pm 2.1$ & $17.5 \pm 2.1$ & $17.4 \pm 1.8$ & $17.6 \pm 2.0$ & $17.3 \pm 2.1$ & $17.4 \pm 2.0$ & $16.8 \pm 1.7^{(n=27)}$ & $\begin{array}{c}-0.005(-0.02 \\
0.02)\end{array}$ & 0.001 & 0.649 & $\begin{array}{l}0.03(0.01 \\
0.05)\end{array}$ & 0.41 & 0.001 \\
\hline
\end{tabular}

Values are mean $\pm S D$.

Bold indicates significance.

Adjustments were made for weight and height.

$\beta$-Coefficients are calculated with age as a continuous variable.

Superscript values indicate the group numbers.

$a B M D$, areal bone mineral density; $B M C$, bone mineral content; $B A$, bone area. 
TABLE 6 | Dual energy X-ray absorptiometry bone parameters in women.

\begin{tabular}{|c|c|c|c|c|c|c|c|c|c|c|c|c|c|c|}
\hline Age (yr) & $\begin{array}{c}\mathbf{4 0 - 4 4} \\
(n=28)\end{array}$ & $\begin{array}{c}45-49 \\
(n=32)\end{array}$ & $\begin{array}{l}50-54 \\
(n=30)\end{array}$ & $\begin{array}{l}\mathbf{5 5 - 5 9} \\
(n=31)\end{array}$ & $\begin{array}{l}60-64 \\
(n=31)\end{array}$ & $\begin{array}{c}65-69 \\
(n=33)\end{array}$ & $\begin{array}{c}70-74 \\
(n=30)\end{array}$ & $\begin{array}{c}75+ \\
(n=34)\end{array}$ & $\begin{array}{c}\beta \text {-Coefficient } \\
(95 \% \mathrm{Cl})\end{array}$ & $R^{2}$ & $\begin{array}{c}\text { Unadjusted } \\
p \text {-value }\end{array}$ & $\begin{array}{c}\beta \text {-Coefficient } \\
(95 \% \mathrm{Cl})\end{array}$ & $R^{2}$ & $\begin{array}{l}\text { Adjusted } \\
p \text {-value }\end{array}$ \\
\hline \multicolumn{15}{|c|}{ Whole body } \\
\hline $\begin{array}{l}\mathrm{aBMD} \\
\left(\mathrm{g} / \mathrm{cm}^{2}\right)\end{array}$ & $1.1 \pm 0.1^{(n=27)}$ & $1.1 \pm 0.1$ & $1.0 \pm 0.1$ & $1.0 \pm 0.1^{(n=30)}$ & $1.0 \pm 0.1$ & $0.9 \pm 0.1$ & $0.9 \pm 0.1^{(n=29)}$ & $0.9 \pm 0.1^{(n=30)}$ & $\begin{array}{l}-0.005(-0.006 \\
-0.005)\end{array}$ & 0.37 & $<0.0001$ & $\begin{array}{l}-0.004 \\
(-0.005 \\
-0.003)\end{array}$ & 0.54 & $<0.0001$ \\
\hline $\begin{array}{l}\text { BMC } \\
\text { (g) }\end{array}$ & $2,273 \pm 361^{(n=27)}$ & $2,210 \pm 366$ & $2,081 \pm 449$ & $1,827 \pm 300^{(n=30)}$ & $1,752 \pm 252$ & $1,749 \pm 369$ & $1,679 \pm 368^{(n=29)}$ & $1,538 \pm 303^{(n=30)}$ & $\begin{array}{l}-18.9(-22.5 \\
-15.3)\end{array}$ & 0.31 & $<0.0001$ & $\begin{array}{c}-10.6 \\
(-12.9 \\
-8.2)\end{array}$ & 0.75 & $<0.0001$ \\
\hline $\begin{array}{l}\mathrm{BA} \\
\left(\mathrm{cm}^{2}\right)\end{array}$ & $2,025 \pm 193^{(n=27)}$ & $2,041 \pm 229$ & $1,974 \pm 250$ & $1,840 \pm 201^{(n=30)}$ & $1,820 \pm 165$ & $1,839 \pm 241$ & $1,781 \pm 231^{(n=29)}$ & $1,703 \pm 227^{(n=30)}$ & $\begin{array}{l}-8.7(-11.0 \\
-6.5)\end{array}$ & 0.20 & $<0.0001$ & $\begin{array}{c}--2.8(-4.0 \\
-1.7)\end{array}$ & 0.83 & $<0.0001$ \\
\hline \multicolumn{15}{|l|}{ Spine } \\
\hline $\begin{array}{l}\text { aBMD } \\
\left(\mathrm{g} / \mathrm{cm}^{2}\right)\end{array}$ & $1.1 \pm 0.1^{(n=27)}$ & $1.0 \pm 0.1$ & $0.9 \pm 0.2$ & $0.8 \pm 0.1^{(n=30)}$ & $0.8 \pm 0.1$ & $0.8 \pm 0.2^{(n=32)}$ & $0.8 \pm 0.1^{(n=29)}$ & $0.7 \pm 0.1^{(n=31)}$ & $\begin{array}{l}-0.008(-0.01 \\
-0.007)\end{array}$ & 0.34 & $<0.0001$ & $\begin{array}{l}-0.006 \\
(-0.008 \\
-0.005)\end{array}$ & 0.50 & $<0.0001$ \\
\hline $\begin{array}{l}\text { BMC } \\
\text { (g) }\end{array}$ & $53.3 \pm 9.5^{(n=27)}$ & $48.7 \pm 10.1$ & $45.5 \pm 10.1$ & $37.6 \pm 8.1^{(n=30)}$ & $38.2 \pm 7.2$ & $37.6 \pm 10.2^{(n=32)}$ & $35.7 \pm 9.2^{(n=29)}$ & $32.9 \pm 8.5^{(n=31)}$ & $\begin{array}{l}-0.5(-0.6 \\
-0.4)\end{array}$ & 0.29 & $<0.0001$ & $\begin{array}{c}-0.3(-0.4 \\
-0.2)\end{array}$ & 0.58 & $<0.0001$ \\
\hline $\begin{array}{l}\mathrm{BA} \\
\left(\mathrm{cm}^{2}\right)\end{array}$ & $48.6 \pm 3.6^{(n=27)}$ & $48.5 \pm 4.9$ & $48.5 \pm 4.4$ & $46.4 \pm 5.4^{(n=30)}$ & $46.9 \pm 4.3$ & $47.7 \pm 5.2^{(n=32)}$ & $45.6 \pm 5.1^{(n=29)}$ & $44.9 \pm 6.1^{(n=31)}$ & $\begin{array}{l}-0.1(-0.2 \\
-0.1)\end{array}$ & 0.07 & $<0.0001$ & $\begin{array}{c}-0.007 \\
(-0.05 \\
0.03)\end{array}$ & 0.47 & 0.734 \\
\hline \multicolumn{15}{|c|}{ Total hip } \\
\hline $\begin{array}{l}\mathrm{aBMD} \\
\left(\mathrm{g} / \mathrm{cm}^{2}\right)\end{array}$ & $1.0 \pm 0.2^{(n=27)}$ & $1.0 \pm 0.1^{(n=30)}$ & $0.9 \pm 0.1$ & $0.8 \pm 0.1^{(n=30)}$ & $0.8 \pm 0.1^{(n=29)}$ & $0.7 \pm 0.1^{(n=32)}$ & $0.7 \pm 0.1^{(n=29)}$ & $0.7 \pm 0.1^{(n=33)}$ & $\begin{array}{l}-0.009(-0.01 \\
-0.008)\end{array}$ & 0.44 & $<0.0001$ & $\begin{array}{l}-0.008 \\
(-0.009 \\
-0.006)\end{array}$ & 0.51 & $<0.0001$ \\
\hline $\begin{array}{l}\text { BMC } \\
\text { (g) }\end{array}$ & $28.6 \pm 5.0^{(n=27)}$ & $28.1 \pm 4.8^{(n=30)}$ & $25.0 \pm 4.5$ & $23.0 \pm 3.3^{(n=30)}$ & $22.0 \pm 3.2^{(n=29)}$ & $21.9 \pm 3.6^{(n=32)}$ & $20.9 \pm 4.4^{(n=29)}$ & $19.2 \pm 3.9^{(n=33)}$ & $\begin{array}{l}-0.2(-0.3 \\
-0.2)\end{array}$ & 0.35 & $<0.0001$ & $\begin{array}{c}-0.2(-0.2 \\
-0.1)\end{array}$ & 0.51 & $<0.0001$ \\
\hline $\mathrm{BA}\left(\mathrm{cm}^{2}\right)$ & $28.3 \pm 2.1^{(n=27)}$ & $28.5 \pm 1.9^{(n=30)}$ & $28.8 \pm 2.1$ & $28.3 \pm 2.0^{(n=30)}$ & $28.5 \pm 1.9^{(n=29)}$ & $29.6 \pm 2.3^{(n=32)}$ & $28.4 \pm 2.3^{(n=29)}$ & $28.6 \pm 2.3^{(n=33)}$ & $\begin{array}{l}0.004(-0.02 \\
0.03)\end{array}$ & 0.0005 & 0.719 & $\begin{array}{l}0.04(0.02 \\
0.06)\end{array}$ & 0.39 & $<0.0001$ \\
\hline \multicolumn{15}{|c|}{ Femoral neck } \\
\hline $\begin{array}{l}\mathrm{aBMD} \\
\left(\mathrm{g} / \mathrm{cm}^{2}\right)\end{array}$ & $1.0 \pm 0.1^{(n=27)}$ & $1.0 \pm 0.1^{(n=30)}$ & $0.9 \pm 0.1$ & $0.8 \pm 0.1^{(n=30)}$ & $0.8 \pm 0.1^{(n=29)}$ & $0.7 \pm 0.1^{(n=32)}$ & $0.7 \pm 0.1^{(n=29)}$ & $0.7 \pm 0.1^{(n=32)}$ & $\begin{array}{l}-0.008(-0.01 \\
-0.007)\end{array}$ & 0.45 & $<0.0001$ & $\begin{array}{l}-0.007 \\
(-0.008 \\
-0.006)\end{array}$ & 0.54 & $<0.0001$ \\
\hline $\begin{array}{l}\text { BMC } \\
\text { (g) }\end{array}$ & $4.1 \pm 0.8^{(n=27)}$ & $4.0 \pm 0.8^{(n=30)}$ & $3.6 \pm 0.8$ & $3.3 \pm 0.7^{(n=30)}$ & $3.2 \pm 0.6^{(n=29)}$ & $3.1 \pm 0.7^{(n=32)}$ & $3.0 \pm 0.7^{(n=29)}$ & $2.6 \pm 0.7^{(n=32)}$ & $\begin{array}{l}-0.04(-0.04 \\
-0.03)\end{array}$ & 0.29 & $<0.0001$ & $\begin{array}{l}-0.03 \\
(-0.03 \\
-0.02)\end{array}$ & 0.44 & $<0.0001$ \\
\hline $\begin{array}{l}\mathrm{BA} \\
\left(\mathrm{cm}^{2}\right)\end{array}$ & $4.2 \pm 0.7^{(n=27)}$ & $4.1 \pm 0.6^{(n=30)}$ & $4.2 \pm 0.6$ & $4.1 \pm 0.7^{(n=30)}$ & $4.1 \pm 0.6^{(n=29)}$ & $4.2 \pm 0.7^{(n=32)}$ & $4.2 \pm 0.6^{(n=29)}$ & $3.9 \pm 0.7^{(n=32)}$ & $\begin{array}{l}-0.005 \text { (-0.01, } \\
0.002)\end{array}$ & 0.01 & 0.124 & $\begin{array}{c}0.001 \\
(-0.006 \\
0.008)\end{array}$ & 0.14 & 0.775 \\
\hline \multicolumn{15}{|l|}{ Radius } \\
\hline $\begin{array}{l}\mathrm{aBMD} \\
\left(\mathrm{g} / \mathrm{cm}^{2}\right)\end{array}$ & $0.6 \pm 0.1^{(n=27)}$ & $0.6 \pm 0.1$ & $0.6 \pm 0.1$ & $0.5 \pm 0.1^{(n=29)}$ & $0.5 \pm 0.1$ & $0.4 \pm 0.1$ & $0.5 \pm 0.1$ & $0.4 \pm 0.1^{(n=32)}$ & $\begin{array}{l}-0.006(-0.007 \\
-0.005)\end{array}$ & 0.44 & $<0.0001$ & $\begin{array}{l}-0.005 \\
(-0.005 \\
-0.004)\end{array}$ & 0.58 & $<0.0001$ \\
\hline $\begin{array}{l}\text { BMC } \\
\text { (g) }\end{array}$ & $9.0 \pm 1.5^{(n=27)}$ & $8.8 \pm 1.2$ & $8.3 \pm 1.8$ & $7.0 \pm 1.2^{(n=29)}$ & $7.1 \pm 1.3$ & $6.8 \pm 1.6$ & $6.6 \pm 1.4$ & $6.1 \pm 1.2^{(n=32)}$ & $\begin{array}{l}-0.08(-0.09 \\
-0.06)\end{array}$ & 0.31 & $<0.0001$ & $\begin{array}{c}-0.05(-0.06 \\
-0.04)\end{array}$ & 0.60 & $<0.0001$ \\
\hline $\begin{array}{l}\mathrm{BA} \\
\left(\mathrm{cm}^{2}\right)\end{array}$ & $14.5 \pm 1.3^{(n=27)}$ & $15.0 \pm 1.7$ & $14.7 \pm 1.8$ & $14.8 \pm 1.5^{(n=29)}$ & $15.0 \pm 1.6$ & $15.6 \pm 1.2$ & $14.7 \pm 1.5$ & $15.3 \pm 1.9^{(n=32)}$ & $\begin{array}{l}0.01(-0.003, \\
0.03)\end{array}$ & 0.01 & 0.103 & $\begin{array}{l}0.04(0.03 \\
0.05)\end{array}$ & 0.41 & $<0.0001$ \\
\hline
\end{tabular}

Values are mean $\pm S D$

Bold indicates significance.

Adjustments were made for weight and height.

$\beta$-Coefficients are calculated with age as a continuous variable.

Superscript values indicate the group numbers.

$a B M D$, areal bone mineral density; $B M C$, bone mineral content; $B A$, bone area. 

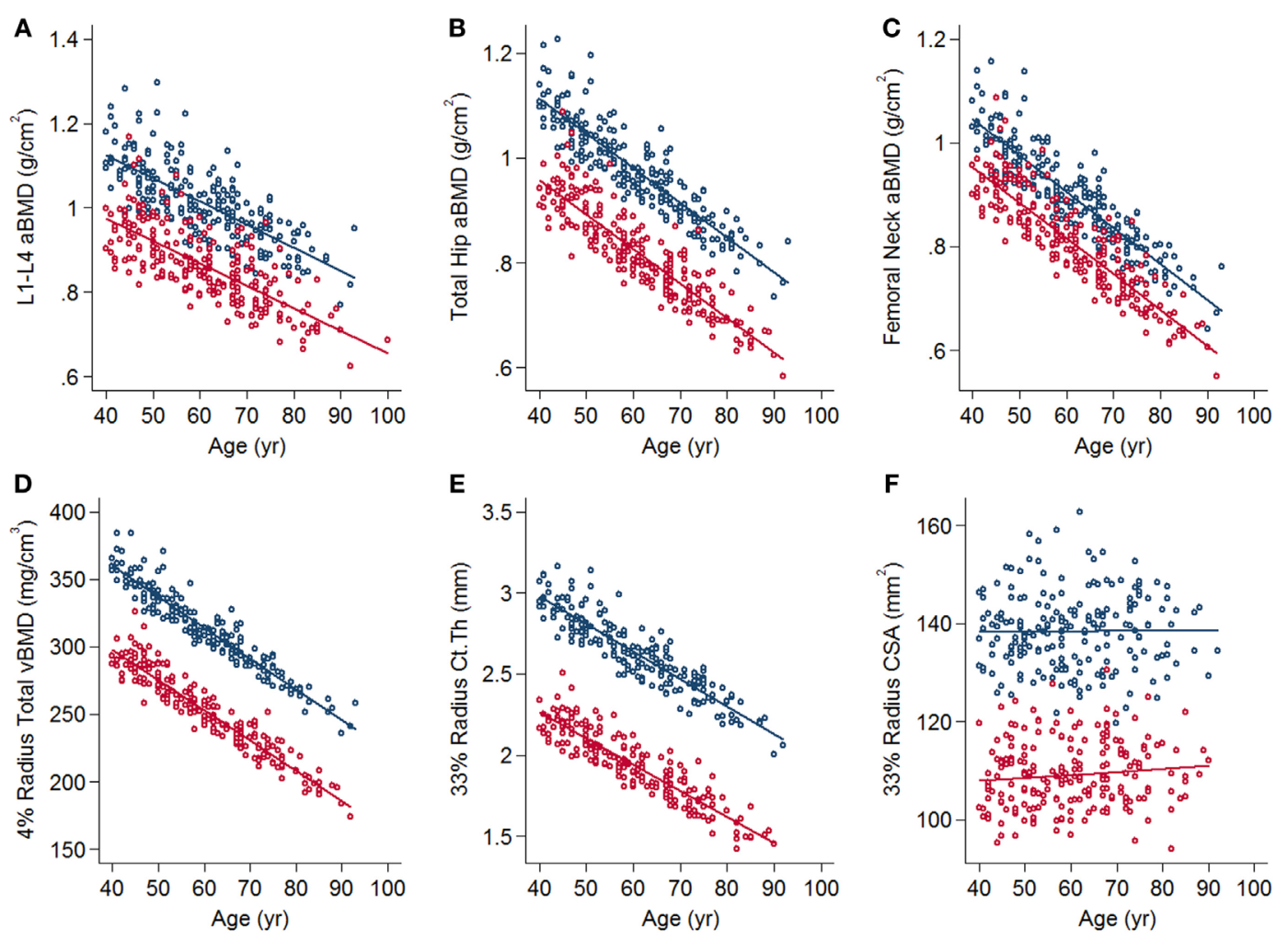

FIGURE 4 | Associations between age and clinically relevant bone outcomes (A) L1-L4 areal bone mineral density (aBMD), (B) total hip aBMD, (C) femoral neck aBMD, (D) 4\% radius total volumetric BMD, (E) 33\% radius cortical thickness, and (F) 33\% radius cross-sectional area. Scatter plots are from linear regression with adjustments for sex, weight, and height. Blue lines and dots represent men and red lines and dots represent women.

Data from pQCT in men and women are shown in Tables 7 and $\mathbf{8}$, respectively. At the distal radius, there were greater negative differences with age in women compared to men in the $4 \%$ total vBMD. At the $33 \%$ cortical site, there was a greater reduction in cortical thickness in women than in men and a concurrent greater decrease in cortical vBMD. There were small, but greater increases in CSA in women (Figures 4D-F). At the $4 \%$ tibia, there were greater negative differences in Trab.vBMD with age in women than in men following adjustments for body size $\left(R^{2}=0.41\right.$, interaction $\left.p<0.0001\right)$.

From the LVA scans, $9 \%$ of GamBAS participants had moderate or severe vertebral fractures (as defined by GE Lunar software), and $14 \%$ had spinal degeneration (osteophytes present). Hip fractures were self-reported; $3 \%$ of women and $0.4 \%$ of men reported a hip fracture, or fracture-like injury. Comparing the GamBAS participants to the manufacturers US Black reference database showed the population to have lower age, and gender-matched $Z$-scores for aBMD than the reference for both the lumbar spine and hip. The mean (SD), range of those $Z$-scores are: lumbar spine L1-L4, women -2.0 (1.1), -4.7 to 1.5 ; men -1.5 (1.3), -4.4 to 3.9 and for femoral neck, women $-1.1(0.9),-3.3$ to 2.0 ; men $-1.0(0.9),-3.2$ to 1.7 . Peripheral vascular calcification in the lower-limb was visible in $16 \%$ of the population. There were no sex differences in the presence of peripheral vascular calcification: $19 \%$ in men vs $15 \%$ in women, $p=0.195$.

\section{DISCUSSION}

GamBAS is the first and largest prospective longitudinal study in West Africa in which quantitative measurements of bone and muscle have been collected. The initial findings have highlighted that women have a greater degree of aBMD loss compared to men, yet have less loss of muscle. Analyses of longitudinal data will allow more accurate quantification of this loss and investigation of the mechanisms driving these effects. Data from DXA demonstrate that height-adjusted BA increased with age at the total hip and radius in both men and women; however, pQCT revealed that the increase in cross-sectional $\mathrm{BA}$ at the radius was only evident in women. The decrease in cortical thickness in women suggests this increase in cross-sectional BA may be a biomechanical adaptation to loss of bone. However, whether this is indicative of a cohort effect or age-related periosteal apposition will be confirmed in analysis of prospective data. In agreement with our previous work, the magnitude of age-differences is similar to that observed in other populations, where fracture incidence is higher.

Our preliminary analyses of cross-sectional data show that total hip aBMD was negatively associated with age in Gambian men and women. Self-reported hip fracture rates were $0.4-3 \%$ and lower compared to sex- and age-adjusted hip fracture incidence elsewhere in the world (32). The aBMD Z-scores we report show 
TABLE 7 | Peripheral quantitative computed tomography bone parameters in men.

\begin{tabular}{|c|c|c|c|c|c|c|c|c|c|c|c|c|c|c|}
\hline Age (yr) & $\begin{array}{l}40-44 \\
(n=25)\end{array}$ & $\begin{array}{l}45-49 \\
(n=34)\end{array}$ & $\begin{array}{l}50-54 \\
(n=29)\end{array}$ & $\begin{array}{l}55-59 \\
(n=31)\end{array}$ & $\begin{array}{l}60-64 \\
(n=27)\end{array}$ & $\begin{array}{l}65-69 \\
(n=35)\end{array}$ & $\begin{array}{l}70-74 \\
(n=30)\end{array}$ & $\begin{array}{c}75+ \\
(n=28)\end{array}$ & $\begin{array}{c}\beta \text {-Coefficient } \\
(95 \% \mathrm{CI})\end{array}$ & $R^{2}$ & $\begin{array}{c}\text { Unadjusted } \\
p \text {-value }\end{array}$ & $\begin{array}{c}\beta \text {-Coefficient } \\
(95 \% \mathrm{CI})\end{array}$ & $R^{2}$ & $\begin{array}{c}\text { Adjusted } \\
p \text {-value }\end{array}$ \\
\hline \multicolumn{15}{|l|}{ Radius } \\
\hline $\begin{array}{l}4 \% \text { Total vBMD } \\
\left(\mathrm{mg} / \mathrm{cm}^{3}\right)\end{array}$ & $362.5 \pm 45.1^{(h=2)}$ & $327.3 \pm 39 . .^{(n=27)}$ & $327.0 \pm 45.0^{(n=25)}$ & $331.9 \pm 56.2^{(n=26)}$ & $296.2 \pm 52.5^{n=249}$ & $292.6 \pm 55.8^{n=25}$ & $276.0 \pm 46.2^{(n=25)}$ & $292.2 \pm 57.5^{n=23 i}$ & $-1.92(-2.5,-1.3)$ & 0.18 & $<0.0001$ & $-1.77(-2.4,-1.2)$ & 0.20 & $<0.0001$ \\
\hline $\begin{array}{l}4 \% \text { Trab.vBMD } \\
\left(\mathrm{mg} / \mathrm{cm}^{3}\right)\end{array}$ & $206.5 \pm 44.7^{(n=22)}$ & $181.6 \pm 39.7 n=27)$ & $173.1 \pm 31.3^{(n=25)}$ & $177.3 \pm 41.8^{n=26}$ & $152.3 \pm 40.2^{(n=24)}$ & $147.9 \pm 38.6^{(n=25)}$ & $149.6 \pm 34.6^{(n=25)}$ & $140.2 \pm 35.2^{(n=23)}$ & $-1.57(-2.0,-1.1)$ & 0.21 & $<0.0001$ & $-1.45(-1.9,-1.0)$ & 0.23 & $<0.0001$ \\
\hline $\begin{array}{l}33 \% \text { Ct.vBMD } \\
\left(\mathrm{mg} / \mathrm{cm}^{3}\right)\end{array}$ & $1,228.9 \pm 35.8^{(n=24)}$ & $1,230.8 \pm 37.8^{n=29}$ & $1,235.0 \pm 33.8^{n=25}$ & $1,224.0 \pm 34.1^{(n=28)}$ & $1,203 \cdot 2 \pm 44 \cdot 9^{(h=23)}$ & $1,203.8 \pm 25.9^{(n=26)}$ & $1,192.5 \pm 30.1^{(n=25)}$ & $1,207.0 \pm 31.4^{(n=23)}$ & $-0.96(-1.4,-0.6)$ & 0.10 & $<0.0001$ & $-0.94(-1.4,-0.5)$ & 0.11 & $<0.0001$ \\
\hline $33 \% \mathrm{CSA}\left(\mathrm{mm}^{2}\right)$ & $139.5 \pm 18.0^{(n=24)}$ & $135.2 \pm 20.2^{h=29 i}$ & $\left.142.8 \pm 20.0{ }^{n}=25\right)$ & $140.9 \pm 16.5^{h(n=28)}$ & $142.6 \pm 17.4^{n=23)}$ & $134.7 \pm 19.3^{n=26 i}$ & $138.8 \pm 20.4^{(n=25)}$ & $133.4 \pm 14.9^{(n=23)}$ & $-0.12(-0.3,0.1)$ & 0.01 & 0.274 & $0.14(-0.1,0.3)$ & 0.19 & 0.188 \\
\hline $33 \%$ Ct.Th $(\mathrm{mm})$ & $2.7 \pm 0.3^{(m=24)}$ & $2.7 \pm 0.3^{(n=29)}$ & $2.8 \pm 0.3^{(n=25)}$ & $2.7 \pm 0.3^{(n=28)}$ & $2.6 \pm 0.3^{(n=23)}$ & $2.6 \pm 0.4^{(n=26)}$ & $2.4 \pm 0.4^{(n=25)}$ & $2.5 \pm 0.3^{(n=23)}$ & $-0.01(-0.01,-0.01)$ & 0.11 & $<0.0001$ & $-0.01(-0.01,-0.003)$ & 0.16 & 0.001 \\
\hline $33 \% \mathrm{SSI}\left(\mathrm{mm}^{3}\right)$ & $310.1 \pm 0.3^{(m=24)}$ & $293.5 \pm 63.0^{(n=29)}$ & $354.2 \pm 80.3^{n=25]}$ & $305.3 \pm 69.8(n=28)$ & $332.6 \pm 61.7^{(n=23)}$ & $297.9 \pm 84.6^{(n=26)}$ & $309.2 \pm 86.0^{(n=25)}$ & $287.9 \pm 51.5^{(n=23)}$ & $-0.60(-1.4,0.2)$ & 0.01 & 0.150 & $0.32(-0.5,1.1)$ & 0.17 & $<0.0001$ \\
\hline \multicolumn{15}{|l|}{ Tibia } \\
\hline $\begin{array}{l}\text { 4\% Total vBMD } \\
\left(\mathrm{mg} / \mathrm{cm}^{3}\right)\end{array}$ & $316.9 \pm 43.7$ & $283.0 \pm 38.3^{n=300}$ & $285.2 \pm 39.2^{(n=25)}$ & $275.8 \pm 37.10=28)$ & $255.1 \pm 42.5^{(n=25)}$ & $259.8 \pm 48.3^{n=32 i}$ & $245.5 \pm 33.00^{(n=27)}$ & $249.9 \pm 40.1^{(n=24)}$ & $-1.62(-2.1,-1.2)$ & 0.19 & $<0.0001$ & $-1.45(-1.9,-1.0)$ & 0.31 & $<0.0001$ \\
\hline $\begin{array}{l}\text { 4\% Trab.vBMD } \\
\left(\mathrm{mg} / \mathrm{cm}^{3}\right)\end{array}$ & $204.4 \pm 40.3^{(n=24)}$ & $\left.186.8 \pm 33.9^{n}=30\right)$ & $\left.181.6 \pm 29.3^{n}=25\right)$ & $172.8 \pm 26.9^{(n=28)}$ & $169.5 \pm 32.2^{(n=25)}$ & $170.0 \pm 37.00^{n=32}$ & $161.2 \pm 25.6^{(n=27)}$ & $163.0 \pm 30.00^{n=24)}$ & $-0.96(-1.3,-0.6)$ & 0.12 & $<0.0001$ & $-0.81(-1.2,-0.5)$ & 0.25 & $<0.0001$ \\
\hline $\begin{array}{l}38 \% \text { Ct.vBMD } \\
\left(\mathrm{mg} / \mathrm{cm}^{3}\right)\end{array}$ & $1,219.8 \pm 33.8$ & $1,218.6 \pm 29.0^{(n=31)}$ & $1,220.1 \pm 37.1^{n=25}$ & $1,218.7 \pm 39.6^{n=28}$ & $1,199.5 \pm 42.6^{(n=26)}$ & $1,204 \cdot 3 \pm 31.0^{n=32}$ & $1,193.6 \pm 35.5^{(n=27)}$ & $1,202.4 \pm 32.1^{(m=24)}$ & $-0.62(-1.0,-0.2)$ & 0.05 & 0.002 & $-0.79(-1.2,-0.4)$ & 0.08 & $<0.0001$ \\
\hline $38 \% \mathrm{CSA}\left(\mathrm{mm}^{2}\right)$ & $472.2 \pm 61.4$ & $461.5 \pm 64.3^{(n=31)}$ & $453.3 \pm 52.2^{(n=25)}$ & $455.4 \pm 39.1^{(h=28)}$ & $462.9 \pm 66.6^{(n=26)}$ & $442.3 \pm 55.1^{(n=32)}$ & $452.5 \pm 63.4^{(n=27)}$ & $425.4 \pm 38.8^{(i=24)}$ & -0.8 & 0.04 & 0.0 & 0.0 & 0.28 & 0.989 \\
\hline $38 \%$ Ct.Th $(\mathrm{mm})$ & $5.0 \pm 0.5$ & $5.0 \pm 0.5^{(n=31)}$ & $5.0 \pm 0.4^{(n=25)}$ & $4.9 \pm 0.5^{(n=28)}$ & $4.6 \pm 0.6^{(n=26)}$ & $4.7 \pm 0.6^{(n=32)}$ & $4.8 \pm 0.7^{n=27}$ & $4.8 \pm 0 . .^{(n=24)}$ & $-0.01(-0.01,-0.001)$ & 0.02 & 0.026 & $-0.004(-0.01,0.002)$ & 0.07 & 0.203 \\
\hline $38 \%$ SSI $\left(\mathrm{mm}^{3}\right)$ & $2,129.2 \pm 360.9$ & $2,043.5 \pm 364.8^{(n=31)}$ & $2,033.3 \pm 320.1 n=25)$ & $2,046.3 \pm 253.2^{(n=28)}$ & $1,928.8 \pm 379.0(n=26)$ & $1,896.7 \pm 314.8^{(n=32)}$ & $1,949.7 \pm 354.9^{m=27}$ & $1,839.5 \pm 212.5^{h=24}$ & $-6.37(-9.9,-2.9)$ & 0.06 & $<0.0001$ & $-1.54(-4.9,1.8)$ & 0.27 & 0.362 \\
\hline
\end{tabular}

Values are mean $\pm S D$.

Bold indicates significance.

Adjustments were made for weight and height

$\beta$-Coefficients are calculated with age as a continuous variab

Superscipt valus ind

TABLE 8 | Peripheral quantitative computed tomography bone parameters in women.

\begin{tabular}{|c|c|c|c|c|c|c|c|c|c|c|c|c|c|c|}
\hline Age (yr) & $\begin{array}{l}40-44 \\
(n=28)\end{array}$ & $\begin{array}{l}45-49 \\
(n=32)\end{array}$ & $\begin{array}{l}50-54 \\
(n=30)\end{array}$ & $\begin{array}{c}55-59 \\
(n=31)\end{array}$ & $\begin{array}{l}60-64 \\
(n=31)\end{array}$ & $\begin{array}{l}65-69 \\
(n=33)\end{array}$ & $\begin{array}{l}70-74 \\
(n=30)\end{array}$ & $\begin{array}{c}75+ \\
(n=34)\end{array}$ & $\begin{array}{c}\beta \text {-Coefficient } \\
(95 \% \mathrm{CI})\end{array}$ & $R^{2}$ & $\begin{array}{c}\text { Unadjusted } \\
p \text {-value }\end{array}$ & $\begin{array}{c}\beta \text {-Coefficient } \\
(95 \% \mathrm{CI})\end{array}$ & $R^{2}$ & $\begin{array}{l}\text { Adjusted } \\
p \text {-value }\end{array}$ \\
\hline \multicolumn{15}{|l|}{ Radius } \\
\hline $\begin{array}{l}4 \% \text { Total vBMD } \\
\left(\mathrm{mg} / \mathrm{cm}^{3}\right)\end{array}$ & $305.9 \pm 46.1^{(n=25)}$ & $294.0 \pm 47.3^{(n=30)}$ & $267.7 \pm 45.5^{n=26]}$ & $245.0 \pm 34.9^{n=25)}$ & $244.9 \pm 43.6^{(n=25)}$ & $214.7 \pm 42.8^{(8=29)}$ & $236.0 \pm 39.1^{(n=22)}$ & $208.1 \pm 39.11^{n=30)}$ & $-2.57(-3.0,-2.1)$ & 0.36 & $<0.0001$ & $-2.31(-2.8,-1.8)$ & 0.41 & $<0.0001$ \\
\hline $\begin{array}{l}\text { 4\% Trab.vBMD } \\
\left(\mathrm{mg} / \mathrm{cm}^{3}\right)\end{array}$ & $146.5 \pm 37.0^{(n=25)}$ & $144.5 \pm 33.8^{(r=30)}$ & $122.3 \pm 30.3^{n=26]}$ & $114.4 \pm 27 . g^{h=25)}$ & $106.5 \pm 21.0^{(n=25)}$ & $97.8 \pm 30.8^{(n=29)}$ & $104.0 \pm 16.8^{n=22)}$ & $83.9 \pm 29.9 n=30$ & $-1.63(-1.9,-1.3)$ & 0.33 & $<0.0001$ & $-1.52(-1.9,-1.2)$ & 0.35 & $<0.0001$ \\
\hline $\begin{array}{l}33 \% \text { Ct.vBMD } \\
\left(\mathrm{mg} / \mathrm{cm}^{3}\right)\end{array}$ & $1,252.2 \pm 39.8^{n=260}$ & $1,235.8 \pm 46.4^{(n=29)}$ & $1,208 \cdot 3 \pm 52.8^{(n=28)}$ & $1,169.4 \pm 46.6^{n=2 \pi}$ & $1,150.3 \pm 35.7^{(n=25)}$ & $1,142.9 \pm 52.5^{(n=28)}$ & $1,154.0 \pm 37.5^{h=22)}$ & $1,131.5 \pm 45.5^{(n=25)}$ & $-3.28(-3.8,-2.7)$ & 0.42 & $<0.0001$ & $-3.03(-3.6,-2.5)$ & 0.45 & $<0.0001$ \\
\hline $33 \% \operatorname{CSA}\left(\mathrm{mm}^{2}\right)$ & $104.8 \pm 11.9^{n=26 i}$ & $109.0 \pm 11.7^{(m=28)}$ & $108.8 \pm 16.6^{(n=28)}$ & $108.9 \pm 17.1 .^{n=27}$ & $109.6 \pm 11.7^{(n=25)}$ & $111.0 \pm 11.7^{(n=28)}$ & $\left.105.5 \pm 13.0^{n}=22\right)$ & $115.6 \pm 16.7^{(n=25)}$ & $0.18(0.02,0.3)$ & 0.02 & 0.027 & $0.33(0.2,0.5)$ & 0.23 & $<0.0001$ \\
\hline $33 \%$ Ct.Th $(\mathrm{mm})$ & $2.4 \pm 0.3^{(n=26)}$ & $2.3 \pm 0.3^{n=29)}$ & $2.2 \pm 0.4^{(n=28)}$ & $1.8 \pm 0.3^{(n=2 \pi}$ & $1.8 \pm 0.3^{(n=25)}$ & $1.7 \pm 0.4^{(n=28)}$ & $1.7 \pm 0.4(n=22)$ & $1.5 \pm 0.3^{(n=25)}$ & $-0.02(-0.03,-0.02)$ & 0.43 & $<0.0001$ & $-0.02(-0.02,-0.02)$ & 0.50 & $<0.0001$ \\
\hline $33 \% \mathrm{SSI}\left(\mathrm{mm}^{3}\right)$ & $215.5 \pm 37.44^{n=26)}$ & $222.7 \pm 34.8^{(n=28)}$ & $211.6 \pm 53.3^{(m=28)}$ & $189.0 \pm 37 .(n n=27)$ & $194.4 \pm 34.5^{(n=25)}$ & $193.7 \pm 126.2^{h=28)}$ & $\left.185.8 \pm 33.3^{n}=22\right\}$ & $191.0 \pm 43 .(1 n=25)$ & $-0.89(-1.3,-0.4)$ & 0.07 & $<0.0001$ & $-0.39(-0.8,0.01)$ & 0.29 & 0.06 \\
\hline \multicolumn{15}{|l|}{ Tibia } \\
\hline $\begin{array}{l}4 \% \text { Total vBMD } \\
\left(\mathrm{mg} / \mathrm{cm}^{3}\right)\end{array}$ & $290.1 \pm 36.0^{(n=26)}$ & $276.5 \pm 38.9^{n=30)}$ & $255.5 \pm 36.8^{(n=30)}$ & $225.5 \pm 29.4^{n=28)}$ & $224.2 \pm 30.4^{(n=27)}$ & $201.1 \pm 35 . .^{(n=31)}$ & $206.6 \pm 38.2^{(n=25)}$ & $187.2 \pm 36.7^{(1(n=31)}$ & $-2.72(-3.1,-2.3)$ & 0.47 & $<0.0001$ & $-2.5(-2.9,-2.1)$ & 0.55 & $<0.0001$ \\
\hline $\begin{array}{l}4 \% \text { Trab.vBMD } \\
\left(\mathrm{mg} / \mathrm{cm}^{3}\right)\end{array}$ & $195.5 \pm 28.6^{n=26)}$ & $186.3 \pm 33.6^{(n=30)}$ & $163.9 \pm 32 . .^{(0 n=30)}$ & $141.0 \pm 32.1^{h=28)}$ & $139.5 \pm 25 . .^{(n=27)}$ & $133.8 \pm 34 . .^{(n=31)}$ & $137.6 \pm 30.9^{(n=25)}$ & $119.0 \pm 31.6^{(n=31)}$ & $-1.90(-2.2,-1.57)$ & 0.35 & $<0.0001$ & $-1.65(-2.0,-1.3)$ & 0.43 & $<0.0001$ \\
\hline $\begin{array}{l}38 \% \text { Ct.vBMD } \\
\left(\mathrm{mg} / \mathrm{cm}^{3}\right)\end{array}$ & $1,234.9 \pm 43.4^{(n=26)}$ & $1,215 \cdot 1 \pm 49 \cdot 9^{(n=20)}$ & $1,197.3 \pm 46.2^{(n=300}$ & $1,161.8 \pm 44.8^{h=20}$ & $1,145.0 \pm 49.0^{(h=27)}$ & $1,144.4 \pm 53.8^{(n=3)}$ & $1,147.7 \pm 47.0^{(n=25)}$ & $1,128.2 \pm 55.3^{(n=31)}$ & $-2.64(-3.2,-2.1)$ & 0.29 & $<0.0001$ & $-2.45(-3.0,-1.9)$ & 0.31 & $<0.0001$ \\
\hline $38 \% \operatorname{CSA}\left(\mathrm{mm}^{2}\right)$ & $362.0 \pm 49.7^{n=26)}$ & $363.2 \pm 43.1^{n=30)}$ & $355.5 \pm 48.5^{n}$ & $363.8 \pm 4$ & $359.1 \pm 35.7^{(n=27)}$ & $373.8 \pm 56$. & $334.5 \pm 46.4^{n=25]}$ & $360.4 \pm 52.7^{(n=31)}$ & & 0.01 & 0.53 & & 0.22 & 0.050 \\
\hline $38 \%$ Ct.Th (mm) & $4.1 \pm 0.6^{(n=26)}$ & $4.0 \pm 0.6^{n=30}$ & $3.9 \pm 0.4^{(n=30)}$ & $3.7 \pm 0.5^{(n=28)}$ & $3.5 \pm 0.6^{n=2 \pi}$ & $3.6 \pm 0.7^{(n=31)}$ & $3.3 \pm 0.7^{(n=25)}$ & $3.0 \pm 0.6^{(n=31)}$ & $3,-0.02)$ & 0.23 & $<0.0001$ & $-0.02(-0.03,-0.01)$ & 0.33 & $<0.0001$ \\
\hline $38 \% \mathrm{SSI}\left(\mathrm{mm}^{3}\right)$ & $1,430.8 \pm 271 \cdot 2^{(n=28)}$ & $1,359.2 \pm 226.1^{h=30}$ & $1,353.3 \pm 283.8^{l n=30}$ & $1,350.1 \pm 224.8^{(n=28)}$ & $1,244.8 \pm 146.2^{(n=2 \pi)}$ & $1,338.9 \pm 313.4^{h n=31)}$ & $1,134.5 \pm 250.5^{n=25]}$ & $1,175.1 \pm 227.5^{(n=31)}$ & $-6.34(-9.0,-3.7)$ & 0.09 & $<0.0001$ & $-2.55(-5.0,-0.1)$ & 0.34 & 0.038 \\
\hline
\end{tabular}

Values are mean $\pm S D$.

Bold indicates significance.

Adjustments were made for weight and height.

$\beta$-Coefficients are calculated with age as a cor

VBMD, volumetric bone mineral density; Trab, trabecular; Ct, cortical; Th, thickness; CSA, cross-sectional area; SSI, stress strain index 
that Gambian men and women had osteoporosis at the lumbar spine compared to the manufacturer's reference US Black population. However, this population may not be the most appropriate one to use as a reference and may have led to overestimating the prevalence of osteoporosis in the population $(33,34)$. Using the LVA scans, for the first time we report the prevalence of vertebral fractures in this cohort as $9 \%$, with $6 \%$ in men and 3\% in women. The prevalence of vertebral fractures among GamBAS participants is consistent with a recent report in South African women, where prevalence was 9 and 5\% in black and white women, respectively (35). The positive association between spine BA and age in men may be due to spinal degeneration artefact and requires further investigation.

Calcium is one of the main mineral components of the skeletal system, and adequate dietary intake is essential for healthy bones. In adults from high-income countries, low calcium intake has been associated with an increased risk of osteoporosis, fractures, and falls (36). We report that habitual dietary calcium intake across the age bands in Gambian adults was low relative to international recommendations, for example in the UK the reference nutrient intake for adults is $700 \mathrm{mg} /$ day (37) and in the US and Canada $1,000-1,200 \mathrm{mg} / \mathrm{d}$ (38). Furthermore, dietary calcium intake was low in Gambians compared to UK adults (743-912 mg/day) (39) and American adults (748-1,209 mg/day) (40). Future analyses of longitudinal data investigating the relationship between dietary intake and musculoskeletal health in Gambian men and women are therefore warranted. It is important to note that in our groups other work in children and women of reproductive age, increasing calcium intake did not have a lasting benefit, and findings contradict those that we would expect in countries where habitual intakes are much higher (18, 41-45).

The strengths and potential limitations of GamBAS merit consideration. This was the first study of ageing in The Gambia and we did not know how well the study would be accepted by the older community in Kiang West. We achieved our sample target within each of our age-stratified groups; the stratified study design ensured equal distribution of sampling across the age bands of men and women recruited. Our suite of measurements is unique and allows detailed characterisation of musculoskeletal outcomes. The long-standing relationship between the population of Kiang West and MRC Keneba is maintained through high levels of communication and interaction with a "researchfriendly" population. The KWDSS information dates back to the 1950 's so that accurate dates of birth for determination of age in the older-age bands is possible and also facilitated study design and recruitment stratification by age and village.

We could not accurately assess menopausal status from the women's health questionnaire as some questions did not translate well in the local language leading to errors in information. Fertility status is sensitive in The Gambian culture and so future studies investigating how best to determine menopausal status are required. It was not possible to distinguish secular differences in attained adult size (older men and women were born at a time when early growth faltering may have been more severe) from age-related changes from the reported cross-sectional data. However, this will be possible with the longitudinal data. The majority of recruits were from the four core villages which may be healthier than the other villages as they are located closer to MRC Keneba where the residents are able to receive medical care. This, and that we only needed to recruit $30 \%$ of the Kiang West residents, to achieve our target sample size, may cause bias in our findings. In The Gambia, traditional bone-setters tend to be used for the treatment of musculoskeletal disorders, including fractures, and the information is not added to clinic medical records. Therefore, the hip fracture data obtained are based on self-report and may be inaccurate or incomplete because verification through radiograph reports is not possible. The vertebral fracture data are from GE Lunar Prodigy scans; in later follow-up using the higher-resolution iDXA, we will be able to more accurately assess vertebral fractures. There was difficulty in obtaining medical records from the participants who were not living in the four core villages of Kiang West. This is a homogenous population with little variance in lifestyle characteristics. When comparing to other regions, sub-Saharan Africa or the globe, it will be important to investigate further the effects of environment such as physical activity levels and occupation. Finally, Kiang West is one region of The Gambia and the data may not be generalizable to urban areas of the country, to other West African countries or to the remainder of the continent.

In conclusion, the GamBAS cohort provides important information on the aetiology of ageing in a rural sub-Saharan setting. This is crucial as the ageing population increases and with it, NCD burden.

\section{COLLABORATION}

The MRC has a long and successful history of conducting collaborative research and we welcome specific proposals for new collaborations. Data sharing is available through collaborative agreements and initial enquiries should be made to the Principal Investigator Kate Ward (kw@mrc.soton.ac.uk).

\section{ETHICS STATEMENT}

Ethics was obtained from the MRC Unit The Gambia Scientific Co-ordinating Committee (SCC) and joint Gambian Government/ MRC Unit The Gambia Ethics committee (SCC\#1222). All participants provided written informed consent. All procedures were carried out in accordance with the Declaration of Helsinki (28). If any participant had potential health problems identified by fieldworkers during recruitment or by the team during the field visit, they were advised to visit the clinic at MRC Keneba for follow-up and were offered transport. Participants with elevated blood pressure [according to WHO guidelines (29)] were followed up and treated as appropriate. Any other abnormalities were discussed with the study physician who followed-up appropriately. For instance, if musculoskeletal abnormalities were detected on the scan, the participant was referred for radiography at MRC Fajara.

\section{AUTHOR CONTRIBUTIONS}

KW, AP, GG, IS, AF, LJ, and YS designed the study; KW, AF, IS, GG, LJ, and YS contributed to the data collection; AZ and 
KW analysed the data from the study; AZ, KW, AP, IS, and GG interpreted the data from the study; AZ, KW, AF, and AP drafted the manuscript; AZ, KW, AP, GG, IS, AF, LJ, and YS revised the manuscript; and KW gave the final approval of the version to be published.

\section{ACKNOWLEDGMENTS}

We thank the residents from the Kiang West region in The Gambia, for their continuous support and participation over several years. Staff of MRC Keneba, particularly Lamin Jammeh, Musa Jarjou, Bai Lamin Dondeh, Michael Mendy, Mustapha Ceesay, Mariama Jammeh, Fatou Manneh, Isatou Camera and Kissima Sawo as well as other staff from MRC Unit The Gambia; both past and present who were and are involved in GamBAS; Jennifer Woolston from MRC Human Nutrition Research, many

\section{REFERENCES}

1. United Nations Department of Economic and Social Affairs Population Division. World Population Ageing. New York: United Nations (2015).

2. World Health Organization. World Health Statistics 2016: Monitoring Health for the SDGs, Sustainable Development Goals. France: World Health Organization (2016).

3. Hennig BJ, Unger SA, Dondeh BL, Hassan J, Hawkesworth S, Jarjou L, et al. Cohort profile: the kiang west longitudinal population study (KWLPS)-a platform for integrated research and health care provision in rural Gambia. Int J Epidemiol (2017) 46(2):e13. doi:10.1093/ije/dyv206

4. UNAIDS. HIV and AIDS Estimates. Gambia (2015). Available from: http:// www.unaids.org/en/regionscountries/countries/gambia/

5. Ceesay SJ, Casals-Pascual C, Erskine J, Anya SE, Duah NO, Fulford AJ, et al. Changes in malaria indices between 1999 and 2007 in the Gambia: a retrospective analysis. Lancet (2008) 372(9649):1545-54. doi:10.1016/ S0140-6736(08)61654-2

6. Aboderin IAG, Beard JR. Older people's health in sub-Saharan Africa. Lancet (2015) 385(9968):e9-11. doi:10.1016/S0140-6736(14)61602-0

7. Oden A, McCloskey EV, Kanis JA, Harvey NC, Johansson H. Burden of high fracture probability worldwide: secular increases 2010-2040. Osteoporos Int (2015) 26(9):2243-8. doi:10.1007/s00198-015-3154-6

8. Prentice A, Shaw J, Laskey MA, Cole TJ, Fraser DR. Bone mineral content of British and rural Gambian women aged 18-80+ years. Bone Miner (1991) 12(3):201-14. doi:10.1016/0169-6009(91)90033-V

9. Aspray TJ, Prentice A, Cole TJ, Sawo Y, Reeve J, Francis RM. Low bone mineral content is common but osteoporotic fractures are rare in elderly rural Gambian women. J Bone Miner Res (1996) 11(7):1019-25. doi:10.1002/ jbmr.5650110720

10. Daniels ED, Pettifor JM, Schnitzler CM, Moodley GP, Zachen D. Differences in mineral homeostasis, volumetric bone mass and femoral neck axis length in black and white South African women. Osteoporos Int (1997) 7(2):105-12. doi:10.1007/BF01623684

11. Ballane G, Cauley JA, Luckey MM, Fuleihan Gel H. Secular trends in hip fractures worldwide: opposing trends East versus West. J Bone Miner Res (2014) 29(8):1745-55. doi:10.1002/jbmr.2218

12. Gregg EW, Pereira MA, Caspersen CJ. Physical activity, falls, and fractures among older adults: a review of the epidemiologic evidence. J Am Geriatr Soc (2000) 48(8):883-93. doi:10.1111/j.1532-5415.2000.tb06884.x

13. Prentice A. Diet, nutrition and the prevention of osteoporosis. Public Health Nutr (2004) 7(1A):227-43. doi:10.1079/PHN2003590

14. Rizzoli R, Abraham C, Brandi ML. Nutrition and bone health: turning knowledge and beliefs into healthy behaviour. Curr Med Res Opin (2014) 30(1):131-41. doi:10.1185/03007995.2013.847410

15. Schoenmakers I, Ginty F, Jarjou LM, Nigdikar S, Bennett J, Laidlaw A, et al. Interrelation of parathyroid hormone and vitamin D metabolites in adolescents from the UK and the Gambia. J Steroid Biochem Mol Biol (2010) 121(1-2):217-20. doi:10.1016/j.jsbmb.2010.03.012 thanks to you all. We would like to dedicate this manuscript to Fatou Manneh, who was an important member of our fieldwork and scanning team and sadly passed away in 2015 .

\section{FUNDING}

GamBAS is funded by the MRC UK (programs U105960371, U123261351, and MC-A760-5QX00) and the Department for International Development (DFID) under the MRC/DFID Concordat agreement.

\section{SUPPLEMENTARY MATERIAL}

The Supplementary Material for this article can be found online at http://journal.frontiersin.org/article/10.3389/fendo.2017.00219/ full\#supplementary-material.

16. Marshall D, Johnell O, Wedel H. Meta-analysis of how well measures of bone mineral density predict occurrence of osteoporotic fractures. BMJ (1996) 312(7041):1254-9. doi:10.1136/bmj.312.7041.1254

17. Zengin A, Prentice A, Ward KA. Ethnic differences in bone health. Front Endocrinol (2015) 6:24. doi:10.3389/fendo.2015.00024

18. Jarjou LM, Prentice A, Sawo Y, Laskey MA, Bennett J, Goldberg GR, et al. Randomized, placebo-controlled, calcium supplementation study in pregnant Gambian women: effects on breast-milk calcium concentrations and infant birth weight, growth, and bone mineral accretion in the first year of life. Am J Clin Nutr (2006) 83(3):657-66.

19. Sawo Y, Jarjou LM, Goldberg GR, Laskey MA, Prentice A. Bone mineral changes after lactation in Gambian women accustomed to a low calcium intake. Eur J Clin Nutr (2013) 67(11):1142-6. doi:10.1038/ejcn.2013.162

20. Dominguez-Salas P, Moore SE, Cole D, da Costa KA, Cox SE, Dyer RA, et al. DNA methylation potential: dietary intake and blood concentrations of one-carbon metabolites and cofactors in rural African women. Am J Clin Nutr (2013) 97(6):1217-27. doi:10.3945/ajcn.112.048462

21. Fitt E, Cole D, Ziauddeen N, Pell D, Stickley E, Harvey A, et al. DINO (diet in nutrients out) - an integrated dietary assessment system. Public Health Nutr (2015) 18(2):234-41. doi:10.1017/S1368980014000342

22. Orwoll E, Blank JB, Barrett-Connor E, Cauley J, Cummings S, Ensrud K, et al. Design and baseline characteristics of the osteoporotic fractures in men (MrOS) study - a large observational study of the determinants of fracture in older men. Contemp Clin Trials (2005) 26(5):569-85. doi:10.1016/j.cct. 2005.05.006

23. Zengin A, Pye SR, Cook MJ, Adams JE, Wu FC, O'Neill TW, et al. Ethnic differences in bone geometry between White, Black and South Asian men in the UK. Bone (2016) 91:180-5. doi:10.1016/j.bone.2016.07.018

24. Hardcastle SA, Gregson CL, Rittweger J, Crabtree N, Ward K, Tobias JH. Jump power and force have distinct associations with cortical bone parameters: findings from a population enriched by individuals with high bone mass. J Clin Endocrinol Metab (2014) 99(1):266-75. doi:10.1210/jc.2013-2837

25. Rittweger J, Schiessl H, Felsenberg D, Runge M. Reproducibility of the jumping mechanography as a test of mechanical power output in physically competent adult and elderly subjects. J Am Geriatr Soc (2004) 52(1):128-31. doi:10.1111/j.1532-5415.2004.52022.x

26. Siglinsky E, Krueger D, Ward RE, Caserotti P, Strotmeyer ES, Harris TB, et al. Effect of age and sex on jumping mechanography and other measures of muscle mass and function. JMusculoskelet Neuronal Interact (2015) 15(4):301-8.

27. Roberts HC, Denison HJ, Martin HJ, Patel HP, Syddall H, Cooper C, et al. A review of the measurement of grip strength in clinical and epidemiological studies: towards a standardised approach. Age Ageing (2011) 40(4):423-9. doi:10.1093/ageing/afr051

28. World Medical Association. World medical association declaration of Helsinki: ethical principles for medical research involving human subjects. JAMA (2013) 310(20):2191-4. doi:10.1001/jama.2013.281053 
29. World Health Organization. Global Reference List of 100 Core Health Indicators. Luxembourg: World Health Organization (2015).

30. Ahlborg HG, Johnell O, Turner CH, Rannevik G, Karlsson MK. Bone loss and bone size after menopause. N Engl J Med (2003) 349(4):327-34. doi:10.1056/ NEJMoa022464

31. Cauley JA, Wu L, Wampler NS, Barnhart JM, Allison M, Chen Z, et al. Clinical risk factors for fractures in multi-ethnic women: the Women's Health Initiative. J Bone Miner Res (2007) 22(11):1816-26. doi:10.1359/jbmr.070713

32. Aspray TJ. Osteoporosis: A Study in a Rural Gambian Community [MD Thesis]. University of Newcastle upon Tyne (2002).

33. Micklesfield LK, Norris SA, Nelson DA, Lambert EV, van der Merwe L, Pettifor JM. Comparisons of body size, composition, and whole body bone mass between North American and South African children. J Bone Miner Res (2007) 22(12):1869-77. doi:10.1359/jbmr.070727

34. Schousboe JT, Shepherd JA, Bilezikian JP, Baim S. Executive summary of the 2013 international society for clinical densitometry position development conference on bone densitometry. JClin Densitom (2013) 16(4):455-66. doi:10.1016/j.jocd.2013.08.004

35. Conradie M, Conradie MM, Scher AT, Kidd M, Hough S. Vertebral fracture prevalence in black and white South African women. Arch Osteoporos (2015) 10:203. doi:10.1007/s11657-015-0203-x

36. Dawson-Hughes B. Calcium insufficiency and fracture risk. Osteoporos Int (1996) 6(Suppl 3):37-41. doi:10.1007/BF01623763

37. Department of Health - Committee on Medical Aspects of Food Policy. Dietary Reference Values for Food Energy and Nutrients for the United Kingdom. London: Her Majesty's Stationery Office (HMSO) (1991).

38. Ross AC, Manson JE, Abrams SA, Aloia JF, Brannon PM, Clinton SK, et al. The 2011 report on dietary reference intakes for calcium and vitamin D from the Institute of Medicine: what clinicians need to know. J Clin Endocrinol Metab (2011) 96(1):53-8. doi:10.1210/jc.2010-2704

39. Public Health England. National Diet and Nutrition Survey Results from Years 5 and 6 (Combined) of the Rolling Programme (2012/2013-2013/2014). London, UK: Public Health England (2016).
40. Bailey RL, Dodd KW, Goldman JA, Gahche JJ, Dwyer JT, Moshfegh AJ, et al. Estimation of total usual calcium and vitamin D intakes in the United States. J Nutr (2010) 140(4):817-22. doi:10.3945/jn.109.118539

41. Jarjou LM, Sawo Y, Goldberg GR, Laskey MA, Cole TJ, Prentice A. Unexpected long-term effects of calcium supplementation in pregnancy on maternal bone outcomes in women with a low calcium intake: a follow-up study. Am J Clin Nutr (2013) 98(3):723-30. doi:10.3945/ajcn.113.061630

42. Ward K. Musculoskeletal phenotype through the life course: the role of nutrition. Proc Nutr Soc (2012) 71(1):27-37. doi:10.1017/S0029665111003375

43. Ward KA, Cole TJ, Laskey MA, Ceesay M, Mendy MB, Sawo Y, et al. The effect of prepubertal calcium carbonate supplementation on skeletal development in Gambian boys-a 12-year follow-up study. J Clin Endocrinol Metab (2014) 99(9):3169-76. doi:10.1210/jc.2014-1150

44. Dibba B, Prentice A, Ceesay M, Stirling DM, Cole TJ, Poskitt EM. Effect of calcium supplementation on bone mineral accretion in Gambian children accustomed to a low-calcium diet. Am J Clin Nutr (2000) 71(2):544-9.

45. Prentice A, Dibba B, Sawo Y, Cole TJ. The effect of prepubertal calcium carbonate supplementation on the age of peak height velocity in Gambian adolescents. Am JClin Nutr (2012) 96(5):1042-50. doi:10.3945/ajcn. 112.037481

Conflict of Interest Statement: The authors declare that the research was conducted in the absence of any commercial or financial relationships that could be construed as a potential conflict of interest.

Copyright (c) 2017 Zengin, Fulford, Sawo, Jarjou, Schoenmakers, Goldberg, Prentice and Ward. This is an open-access article distributed under the terms of the Creative Commons Attribution License (CC BY). The use, distribution or reproduction in other forums is permitted, provided the original author(s) or licensor are credited and that the original publication in this journal is cited, in accordance with accepted academic practice. No use, distribution or reproduction is permitted which does not comply with these terms. 